\title{
Density Fluctuations during the Early Stages of Polymer Crystallization: An Overview
}

\author{
Jimmy Baert, Peter Van Puyvelde*
}

The present work provides a critical review of polymer crystallization studies using SALS; experimental methods, analysis techniques, observations and their relations with respect to other techniques are discussed. Furthermore, the fact that nucleation might be accompanied by large scale density fluctuations has been investigated for the flow-induced crystallization of iPB. SALS was applied to measure density and orientation fluctuations, whereas complementary results were obtained from optical microscopy. The observations from both crystallization and melting experiments seem to indicate that the detected density fluctuations result from the presence of weakly anisotropic structures, rather than being an indication of densification before the onset of crystallization.

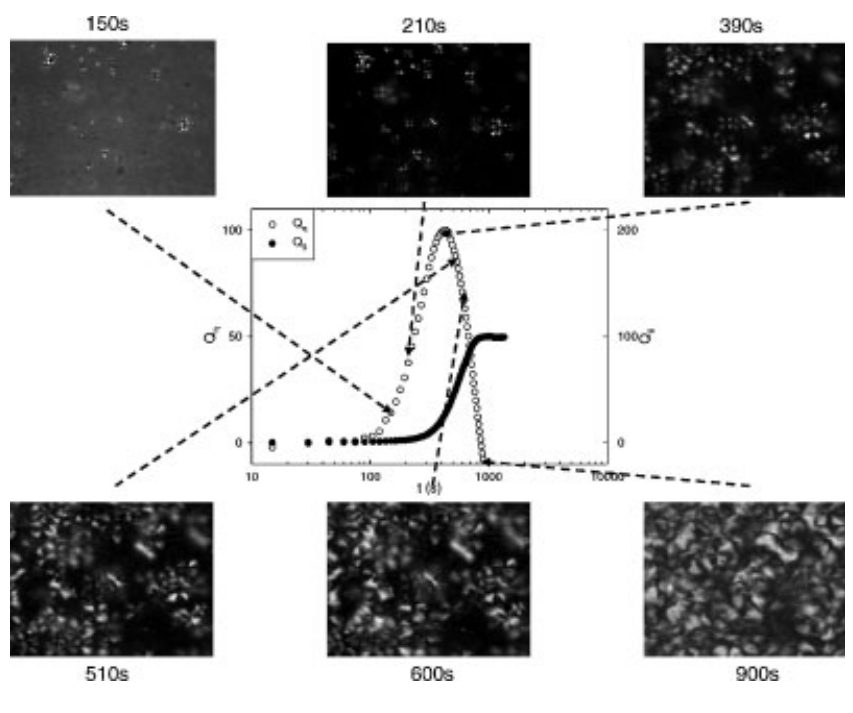

\section{Introduction}

Initial Stages of Polymer Crystallization

Investigations of the kinetics of polymer crystallization are usually carried out with the aid of a wide range of experimental techniques: differential scanning calorimetry (DSC), wide and small angle X-ray scattering (WAXS and SAXS, respectively), dilatometry, small angle light scattering (SALS), microscopy, rheometry, etc. Although based on different properties and probing different physical values to derive information about a sample's crystallinity, all these methods have a similar sensitivity: reliable data can only be obtained for crystallinities

J. Baert, P. Van Puyvelde

Department of Chemical Engineering, Katholieke Universiteit Leuven, W. De Croylaan 46, B-3001, Leuven, Belgium

Fax: +32 1632 2991; E-mail: peter.vanpuyvelde@cit.kuleuven.be

already in the range of some percent of the final value, which is, however, far away from the initial stage of polymer crystallization. Recently, these initial stages have gained particular importance in the discussion of the basic mechanisms of polymer crystallization. The classical nucleation and growth theories for polymer crystallization (e.g., Lauritzen and Hoffman ${ }^{[1]}$ ) do not explain the physical phenomena that are at the origin of the first crystalline entities and they are difficult to validate because the nucleation length scale is not directly accessible with the experimental techniques available at present. In recent years, there have been several experimental observations that led to the assumption that crystallization is preceded by an ordered precursor (so called pre-ordering). Some authors argue there are indications for a nucleation into a mesomorphic phase (e.g., Strobl ${ }^{[2,3]}$ ), while others propose a preceding coverage of the whole volume by a mesomorphic phase which develops by a mechanism resembling a spinodal process (e.g., Olmsted ${ }^{[4]}$ ). However, a commonly 


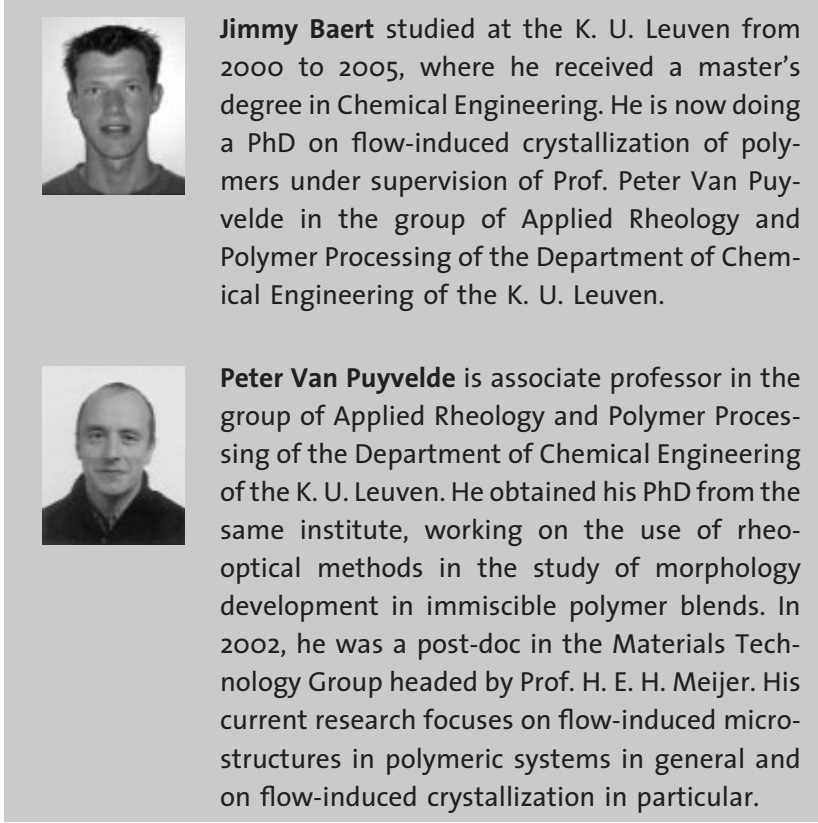

accepted view has not yet evolved. Table 1 lists the most important concepts for polymer crystallization, together with the names of the authors that proposed or used these concepts.

Observations suggesting the presence of a precrystalline, ordered melt have a history extending back for about 40 years. Already in 1967, Katayama et al. ${ }^{[5,6]}$ had observed a SAXS peak significantly earlier than the appearance of crystalline Bragg peaks in the corresponding
WAXS experiment. They proposed that density fluctuations occurred before the formation of any crystals. In the same year, Yeh and Geil ${ }^{[7]}$ reported "ball-like" structures in glassy amorphous poly(ethylene terephthalate) (PET). At that time, the idea of a multistage process was proposed: the authors suggested that these globular structures were composed of clusters of molecules in the glassy state and that lamellae may be formed from planar aggregates of these structures, since their size was comparable to the lamellar thickness. A few years later, Lindenmeyer ${ }^{[8]}$ suggested the idea that polymer crystals grow by deposition of pre-folded chains, and shortly after that, Allegra ${ }^{[9]}$ presented a theory of mesomorphic order in the melt as a precursor to folded chain crystallization, based on the formation of sub-critical nuclei. However, in 1999 the same author ${ }^{[10]}$ refined this theory and proposed a "bundle" model in which the bundles were defined as fluctuating structures with an emerging level of crystallike packing among chain stems. Meanwhile, in 1981, Schultz ${ }^{[11]}$ had introduced a spinodal approach promoting orientation in polymer systems. The difference between these two phase separation mechanisms, spinodal decomposition and nucleation into mesomorphic domains, is that the former is initiated by spontaneous, large range fluctuations in density, without having to pass the energetic barrier corresponding with the creation of a new interface, whereas the latter is initiated by local fluctuations of sufficiently large amplitude which only occur in a small portion of the system - that is, the nuclei.

Table 1. Overview of the most important concepts for polymer crystallization.

\section{Concept}

$1 \quad$ Classical nucleation and growth

Stepwise formation of lamellae by merging of mesomorphic domains
Used by

Lauritzen and Hoffman ${ }^{[1]}$

Wang et al. ${ }^{[19,26]}$

Akpalu et al. ${ }^{[25]}$

Hikosaka et al. ${ }^{\text {[27] }}$

Muthukumar et al. ${ }^{[28,29]}$

Lotz et al. ${ }^{[30,31]}$

Strobl et al. ${ }^{[2,3]}$

Katayama et al. ${ }^{[5,6]}$

Yeh and Geil ${ }^{[7]}$

Lindenmeyer ${ }^{[8]}$

Allegra et al. ${ }^{[9,10]}$

Mirabella et al. ${ }^{[24]}$

Olmsted et al. ${ }^{[4]}$

Schultz et al. ${ }^{[11]}$

Imai et al. ${ }^{[12-15,20-23]}$

Ezquerra et al. ${ }^{[16]}$

Heeley et al. ${ }^{[17,18]}$ 
To verify the proposed mechanisms, many experimental efforts have since been made. Observations of a SAXS signal intensity increase, before the onset of a WAXS signal, during the very early stages of crystallization were reported for PET, ${ }^{[12-15]}$ poly(ether etherketone) (PEEK), ${ }^{[16]}$ isotactic poly(propylene) (iPP) ${ }^{[17,18]}$ and polyethylene $(\mathrm{PE})^{[19]}$ and were claimed to be due to a pre-crystalline orientation in the melt. In some cases it was explained on the basis of the linearized Cahn-Hilliard theory of spinodal decomposition. ${ }^{[13,15,16,20]}$ In another case, ${ }^{[19]}$ the same type of observation was simply attributed to the lower detection limit of crystallinity by SAXS relative to WAXS and not to any pre-crystalline orientation in the melt. This difference in WAXS and SAXS detection sensitivity was claimed to be eliminated in a study by Heeley et al. ${ }^{[18]}$ who used a WAXS detector with a sensitivity many orders of magnitude higher, resulting in the conclusion that a process resembling spinodal decomposition occurs in the early stages of polymer crystallization. In a series of SALS studies on the cold crystallization of PET, ${ }^{[15,21]}$ syndiotactic polystyrene (sPS) ${ }^{[22]}$ and isotactic polystyrene (iPS), ${ }^{[23]}$ the logarithmic increase with time of the orientation fluctuations in the induction period of polymer crystallization could be described by spinodal decomposition kinetics, again supporting the presence of an ordered melt before nucleation. A recent study using atomic force microscopy (AFM) showed that in a branched PE large-scale structures are formed before the formation of any local crystalline structure. ${ }^{[24]}$ In 1998, Olmsted ${ }^{[4]}$ outlined a theory based on spinodal decomposition of a melt, to explain the previous observations of such kinetics in SAXS experiments before the emergence of a crystalline structure. It was thought that conformation ordering, coupled with density fluctuations, is the main event to account for the spinodal decomposition. In the same year, Strobl et al. ${ }^{[2,3]}$ proposed a multistage process to explain polymer crystallization. They describe the process in three steps: formation of weakly oriented mesomorphic domains in the melt, stabilization of this mesomorphic layer by solidification into granular crystal blocks, and merging of the granular blocks into lamellae. Nevertheless, there are also a substantial number of reports that are in contradiction with these stepwise models of polymer crystallization beginning with a mesomorphic melt. Some authors ${ }^{[25-27]}$ reported that no preceding peak in SAXS before WAXS can be observed for PE and some other polymers. Computer simulations of the early stages of crystallization also yielded results in disfavor of a mesomorphic melt and spinodal decomposition of the melt as precursor to crystallization. ${ }^{[28,29]}$ Another strong argument against the concept of a mesomorphic melt was presented by Lotz, ${ }^{[30,31]}$ based on the deposition of helical chain segments of iPP, which were claimed to be permanent markers of the deposition process. The primary conclusion in these studies was that crystallization proceeds by the classical nucleation and growth mechanism and that a mesomorphic melt does not play the role of precursor in polymer crystallization.

Obviously, a clear picture of the initial stages of polymer crystallization is still lacking. Conflicting results favoring various proposed models have been reported, but no convincing conclusions have been reached. The fact that nucleation might be accompanied by large scale fluctuations of the density will be exploited in the present work for shear-induced crystallization of isotactic polybutene (iPB). To understand what controls the structure formation in semi-crystalline polymers, a method to measure and separate density and orientation fluctuations from the microscale and nanoscale spatial arrangement of anisotropic crystals is needed. SALS can be used to provide this information, whereas complementary information can be obtained from polarizing optical microscopy (POM).

\section{SALS in Polymer Crystallization Studies}

Most of the studies using SALS for the study of polymer crystallization are based on the pioneering work of Stein and Wilson, ${ }^{[32]}$ who developed a theory to describe the effects observed for light scattering by a polymer film. The theory is based on a statistical approach which employs correlation functions. During phase transformations the shape, size, internal structure, and order of the scattering units can change. Therefore it was most convenient to describe the scattering in terms of fluctuations. The analysis does not depend on the geometry of the scattering objects but depends only on the contrast causing the scattering. It implies that the scattered intensities are cylindrically symmetric about the incident beam. This is experimentally not found to be true for spherulitic polymers, which do not conform to the assumptions of a random orientation correlation theory. Thus, strictly speaking, the theory can only be applied for objects with isotropic scattering, because it is based on isotropic correlation functions. Although this is rarely the case, in most studies the method is still applied since there are no suitable alternatives. The results - one obtains the mean-squared fluctuations of the density and the local anisotropy - are approximate, but useful.

A more convenient approach to discuss structural changes from scattering measurements, based on the theory of Stein and Wilson, is to use the total integrated scattered intensities (invariants) that are directly proportional to the mean-square fluctuations in refractive index and orientation giving rise to the observed scattering. ${ }^{[3,34]}$ As was already mentioned, the Stein-Wilson approach is adequate only at the early stages of crystallization where 
the approximation of random orientation correlations holds. At later stages, the correlations may become non-random when crystallization occurs through unidirectional growth mechanisms - that is, crystals form lamellae and spherulites. This leads to a non-circular type of scattering, and in this case two-dimensional integration is required for interpretation of the invariants. Despite the development of these approaches in 1974, for the next 20 years SALS was seldom used for the study of polymer crystallization when compared to the other techniques usually employed. The reason for this was twofold: the data evaluation was considered to be difficult, and limitations existed due to the change in sample transparency on crystallization. It was not until the early 90 s before SALS was used more extensively, especially due to the renewed interest in the study of the early stages of crystallization.

In the following paragraphs the most important polymer crystallization studies using SALS are presented. Table 2 gives an overview of these studies. If the results of a study allowed their authors to decide in favor of one of the concepts governing the polymer crystallization (listed in Table 1), this is also mentioned. Okada et al. ${ }^{[35]}$ used SALS in the study of nucleated iPP samples. Analysis of the scattering patterns showed an unexpected difference in onset time between the large scale density and orientation fluctuations; this was ascribed to the development of an isotropic embryo as the precursor of a spherulite. The group of Kaji and Imai complemented DSC, FT-IR spectroscopy and X-ray and neutron scattering experiments with (depolarized) light scattering in order to study the kinetics during the induction period of $\mathrm{PET}{ }^{[15,21,36]} \mathrm{SPS}^{[22]}$ and ${ }_{i P S}{ }^{[23]}$ annealed just above $T_{\mathrm{g}}$. The SALS results showed that the orientation invariant increased exponentially with time, as predicted by the spinodal decomposition theory, suggesting that the parallel ordering of polymer segments actually occurs during the induction period. Pogodina et al. ${ }^{[37]}$ extended the work of Okada et al., ${ }^{[35]}$ and combined SALS experiments with rheological measurements. Again, they observed that orientation fluctuations developed much more slowly and appeared at much later stages in the crystallization process. Based on the evolution of the linear viscoelastic moduli with time and frequency, they suggested that, during the early stages of crystallization, a gel-like structure develops, close to the maximum in density fluctuations and, thus, considerably before crystalline structures appear. Winter et al. ${ }^{[38]}$ were the first to use time-resolved SALS for the study of shearinduced melt crystallization. It was seen that orientation fluctuations in iPP reached saturation at about the time when density fluctuations passed through a maximum, indicating that the system rapidly reached the state of

Table 2. Overview of polymer crystallization studies using SALS.

\begin{tabular}{|c|c|c|c|c|}
\hline Author/Group & Techniques (besides SALS) & Material & Concept & Remarks \\
\hline Okada et al. ${ }^{[35]}$ & - & iPP & 1 & \\
\hline Imai et al. ${ }^{[15,21-23,36]}$ & $\begin{array}{c}\text { SAXS/WAXS, DSC, SANS, } \\
\text { FT-IR, dilatometry }\end{array}$ & $\begin{array}{l}\text { PET; } ;^{[15,21,36]} \\
\text { sPS; }{ }^{[22]} \text { iPS }^{[23]}\end{array}$ & 3 & Cold cryst. \\
\hline Winter $^{[37-39]}$ & $\begin{array}{l}\text { transmitted light, } \\
\text { rheology, DSC, POM }\end{array}$ & iPP & - & Shear flow ${ }^{[38,39]}$ \\
\hline $\operatorname{Devaux}^{[40,41]}$ & $\begin{array}{c}\text { transmitted light, } \\
\text { POM }\end{array}$ & iPP; ; ${ }^{[40,41]} \mathrm{PVDF}^{[41]}$ & 1 & Shear flow \\
\hline Kawai et al. ${ }^{[43]}$ & dilatometry & sPP & - & - \\
\hline Mao et al. ${ }^{[44]}$ & - & PET & 1 & Cold cryst. \\
\hline Fukushima et al. ${ }^{[45-47]}$ & SAXS/WAXS, POM & $\mathrm{PE} ;{ }^{[45,46]} \mathrm{iPP}{ }^{[47]}$ & - & Shear flow \\
\hline Zhang et al. ${ }^{[48]}$ & POM, AFM & iPP & - & Shear flow \\
\hline Somwangthanaroj et al. ${ }^{[49]}$ & POM & iPP/clay nanocomposites & - & Shear flow \\
\hline Xiao et al. ${ }^{[50]}$ & DSC & TiO2/LDPE nanocomposites & - & - \\
\hline Akpalu et al. ${ }^{[51-53]}$ & $\begin{array}{l}\text { transmitted light, } \\
\text { SAXS/WAXS, DSC }\end{array}$ & $\begin{array}{l}\text { ethylene copolymer; }{ }^{[51,53]} \\
\text { PCL/PDLA blend }{ }^{[52]}\end{array}$ & - & $\begin{array}{c}\text { Melting } \\
\text { Behavior }^{[52,53]}\end{array}$ \\
\hline Nishida et al. ${ }^{[54]}$ & POM & PET & 3 & - \\
\hline Wang et al. ${ }^{[55]}$ & POM, TEM & sPS & 3 & Cold cryst. \\
\hline Xiao et al..$^{[56]}$ & SAXS/WAXS & ethylene copolymer & $\begin{array}{l}\text { new concept } \\
\text { required }\end{array}$ & - \\
\hline
\end{tabular}


final crystallinity. For quiescent crystallization, comparison of the SALS data with morphological changes obtained by POM showed that the maximum in density fluctuations occurred close to the instant of spherulitic impingement. In a later study by the same group, ${ }^{[39]}$ the maximum in density fluctuations was used as a characteristic time scale in the investigation of the correlation of material and processing time scales with structure development for shear-induced crystallization of iPP. More recently, Devaux et al. ${ }^{[40,41]}$ used time-resolved SALS experiments for the study of shear-induced crystallization of iPP and PVDF. Their measurements explicitly compare the effect of temperature and shear flow on the kinetics and the intensity of the density fluctuations. They found that temperature and flow have qualitatively similar effects on the time evolution of the density and orientation fluctuations that is, an increase in the relative importance and the kinetics of both invariants with decreasing temperature or increasing shear flow. In a recent paper by JaneschitzKriegl ${ }^{[42]}$ the results of Winter et al. ${ }^{[38]}$ and Devaux et al. ${ }^{[40]}$ are analyzed in terms of the processes occurring during flow-induced crystallization of polymers like iPP. Kawai et al. ${ }^{[43]}$ reported on the crystallization of a sample of sPP. Evaluation of the scattering patterns was used to characterize the development of the size and internal structure of the growing hedrites. Observations pointed toward an in-filling process of crystalline lamellae accompanying the hedrite's growth. Mao et al. ${ }^{[44]}$ monitored the crystallization process of amorphous, glassystate PET by SALS. They showed that large-scale fluctuations happen prior to the crystallization, but the kind of fluctuation is temperature dependent: at the middle temperature, pure density fluctuations take place during the induction period, whereas at lower temperatures, both density and orientation fluctuations occur simultaneously, the latter being the dominant factor. The scattering intensity profiles indicated that the fluctuation behavior for the two conditions resembles the nucleation and growth type phase separation mechanism. Fukushima et al. used time-resolved depolarized light and X-ray scattering to study the structural formation after shear during the early stages of crystallization of both $\mathrm{PE}^{[45,46]}$ and $\mathrm{iPP}^{[47]}$ In order to analyze the SALS patterns, they defined measures for the induction time of the crystallization and the degree of anisotropy of the scattering patterns. It was found that there are two critical shear rates: one for the reduction in the induction time before nucleation, and another for the onset of anisotropy in the scattering patterns. The former critical shear rate is always smaller than the latter one, and both decrease with increasing shear strain. Very recently, Zhang et al. ${ }^{[48]}$ investigated the shear-induced crystallization of iPP at low temperature and low shear rate by in situ optical microscopy, time-resolved SALS, and ex situ AFM in order to explain the formation mechanism of cylindrical structures. They state that, at low temperatures, the core of the cylindrical structure comes from a bundle of shear-stretched strands of an entangled network, with lamellae growing both perpendicular to and along this core.

SALS has also been used in the study of filled polymer and copolymer crystallization. Somwangthanaroj et al. ${ }^{[49]}$ used SALS to study the effect of nanoclay in iPP and Xiao et al. ${ }^{[50]}$ studied the structure of $\mathrm{TiO}_{2} / \mathrm{LDPE}$ nanocomposites. The latter showed that the effects of crystallization conditions and the presence of nanoparticle aggregates on the spherulitic structure of the LDPE matrix can be separated by appropriately analyzing the scattering patterns. Akpalu et al. ${ }^{[51]}$ investigated the crystallization kinetics in a homogeneous copolymer of ethene and 1-octene, combining DSC, SALS, SAXS and WAXS. The difference in onset time between the density and orientation fluctuations and the low azimuthal dependence of the SALS patterns both indicate that, during the early stages of growth, the anisotropy of the growing crystal aggregates is low, suggesting a low crystallinity within the spherulites and/or a low degree of orientational correlations of the constituent crystals. SALS and DSC were also used by Akpalu et al. ${ }^{[52]}$ to characterize the morphology of crystal-amorphous poly(e-caprolactone)/ poly(D,L,lactide) (PCL/PDLA) blends, where temperature variations enabled determination of the onset of meltphase separation. In another study, $\mathrm{Li}$ and Akpalu ${ }^{[53]}$ demonstrated that SALS, in combination with DSC and SAXS, can be used to understand and provide a quantitative description of how crystallization conditions affect the supermolecular structure organization in copolymers of ethylene and alpha-olefins. Nishida et al. ${ }^{[54]}$ observed the emergence of fluctuations with quasi-periodicity, a characteristic of spinodal decomposition, for the crystallization of PET after rapid quenching. Wang et al. ${ }^{[55]}$ extended this work to cold crystallization of sPS, also coming to the conclusion that spinodal phase separation takes place in sPS when heated from the glassy state. Very recently, the structure formation during isothermal crystallization of an ethylene-1-hexene copolymer was probed by light and X-ray scattering. ${ }^{[56]}$ The results showed that the early stage crystallization is characterized by large scale orientation fluctuations that precede the formation of local crystalline order and the initial collapse of these large scale anisotropic/ordered domains. This mechanism could however not be described by the current theoretical models. ${ }^{[3,4,10]}$

From the above overview it is clear that many of the results from SALS studies are contradictory and have raised new questions, some of which will be addressed throughout the rest of this paper. Moreover, the ongoing discussion on the mechanisms that govern the early stages of 
semi-crystalline polymer crystallization confirms the need for further investigations into this domain.

\section{Experimental Part}

\section{Materials}

The isotactic PB-1 (PB0400) used in this study is a commercial grade provided by Basell Polyolefins in the form of pellets. The grade has an isotacticity of $98.8 \%$ and contains no nucleating agents. PB0400 has a $\bar{M}_{\mathrm{W}}=176 \mathrm{~kg} \cdot \mathrm{mol}^{-1}$ and a polydispersity index of 5.7 (data obtained from GPC measurements by Basell Polyolefins). More material properties and a rheological characterization can be found in previous articles. ${ }^{[57,58]}$

\section{Methods}

The optical train used for SALS experiments is presented in Figure 1. Light from a He-Ne laser $(\lambda=632.8 \mathrm{~nm})$ passes successively through a polarizer, the sample contained in a Linkam CSS 450 flow cell, and a Glan-Thompson polarizer. The scattering patterns are collected on a semi-transparent screen. A small rubber cylinder is used as a beamstop. Because the

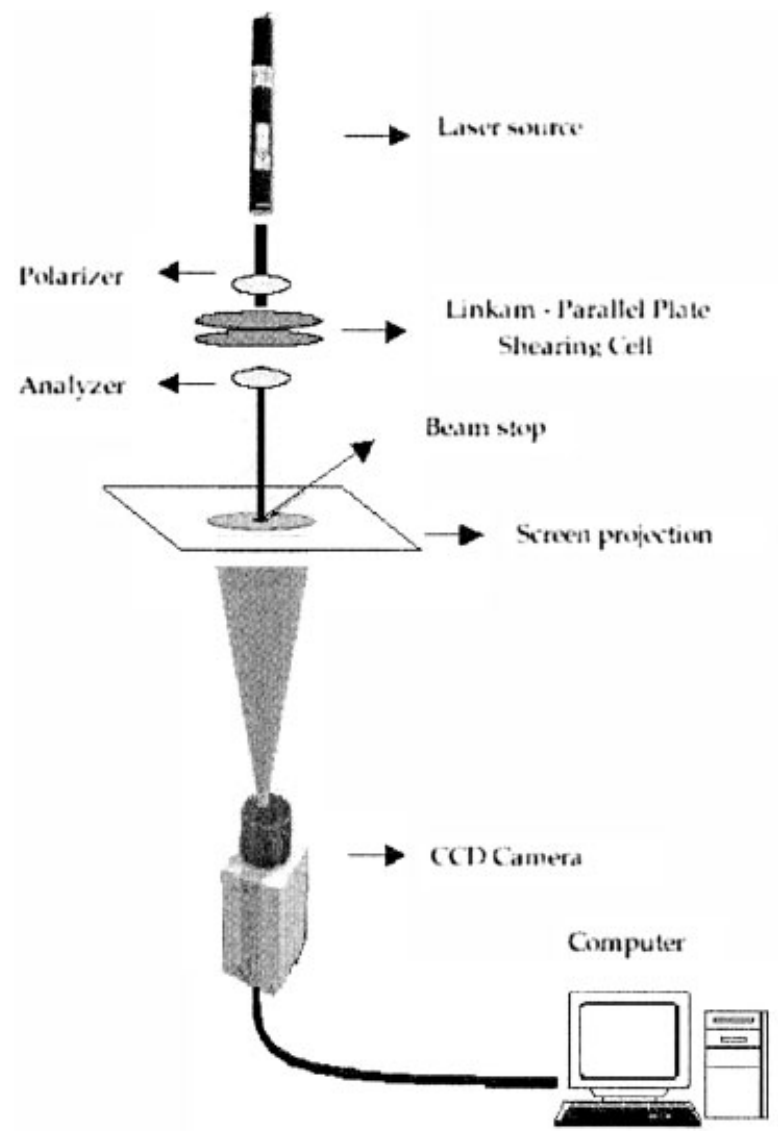

With Frame Grabber on busd nucleation phenomena involve weak signals, a high resolution (10 bits, $1300 \times 1030$ active pixels) light sensitive CCD progressive scan camera (Pulnix TM-1300) was used as the detector. Scattering images were recorded at variable intervals, depending on experimental conditions, and digitized and stored for later analysis. To minimize noise during image processing, a digital frame grabber was used to transfer the images (TCI-digital from Coreco) to a computer. Both polarized scattering experiments, with parallel polarizer and analyzer $\left(V_{\mathrm{v}}\right)$, and depolarized scattering experiments, with crossed polarizers $\left(H_{\mathrm{v}}\right)$, were performed. The samples were sufficiently thin $(0.2 \mathrm{~mm})$ so that loss of scattered energy from the scattered beam through secondary crystallization may be neglected. Home-developed software (SALSsoftware) enables integration over angular quadrants.

The SALS measurements were complemented by direct polarized optical microscopy (POM) observations. To this end, the sample was placed in a Linkam CSS 450 flow cell and observed under a polarizing microscope (Laborlux 12 Pol S, Leitz). The microscope was equipped with a camera (C4742-95, Hamamatsu Photonics) and different magnification objectives. Microscope images were directly digitized by means of a frame grabber (HiPic 6.4.0, Hamamatsu Photonics). Ouantitative analysis of the digital images was carried out by using suitable software (Scion Image, Scion Corp.). The same experimental protocol was used as for the SALS measurements.

\section{Temperature and Shearing Protocol}

The flow-induced isothermal crystallization studies followed the well-known short-term shearing protocol proposed originally by the group of Janeschitz-Kriegl (e.g., ref. ${ }^{[57]}$ ). The thermal protocol was essentially identical in all experiments, whereas the parameters in the shearing protocol were varied systematically. The shearing device was referenced beforehand to its upper position with an initial gap of $h=2500 \mu \mathrm{m}$ and the polymer pellets were inserted between the glass windows of the shearing device. The pellets were pre-heated to $200^{\circ} \mathrm{C}$ and pressed to a thickness $h=210 \mu \mathrm{m}$ by lowering the upper glass plate. The polymer sample was then annealed at $200^{\circ} \mathrm{C}$ for $10 \mathrm{~min}$ to eliminate all possible residual structure and finally cooled at a rate of $10^{\circ} \mathrm{C} \cdot \mathrm{min}^{-1}$ down to the desired crystallization temperature $T_{\mathrm{C}}\left(93,98\right.$ or $\left.103^{\circ} \mathrm{C}\right)$. During cooling, the gap was adjusted in order to compensate for the volumetric shrinkage of the sample that could cause the sample to detach from the plates which could interfere with the optical measurements. The shrinkage occurring during crystallization is not accounted for. It was verified that the mild squeeze flow $\left(10 \mu \mathrm{m}\right.$ at $\left.5 \mu \mathrm{m} \cdot \mathrm{s}^{-1}\right)$ to adjust the gap at $120^{\circ} \mathrm{C}$ did not affect the overall crystallization kinetics. Time zero was assigned to the instant at which the crystallization temperature was reached. Shear flow was then applied at different shear rates, $\dot{\gamma}$, and for different shearing times, $t_{\mathrm{s}}$, with the constraint of a constant applied shear strain: $\gamma=\dot{\gamma} t_{\mathrm{s}}$. The time of shearing was considerably shorter than the crystallization time at $T_{\mathrm{c}}$, so that crystallization during shear was avoided under the conditions studied in this paper. After cessation of shear flow, the samples crystallize isothermally and the crystallization was monitored both by SALS and POM.

Figure 1. SALS setup. 


\section{Analysis of SALS Data}

SALS provides information about fluctuations in density (refractive index) and orientation, which, for semi-crystalline polymers, arise from spatial variations in the crystalline volume fraction and the arrangement of crystalline aggregates within the sample. A beam of polarized light interacts with the microstructure of the crystallizing sample and will be scattered. The scattered light depends on the relative positions of the polarizer and the analyzer (see Figure 1). If the polarizer and analyzer are parallel $\left(V_{\mathrm{V}}\right.$ and $\left.H_{\mathrm{H}}\right)$, the scattering is labeled as polarized, whereas the scattering is called depolarized when the polarizer and analyzer are crossed $\left(H_{\mathrm{V}}\right.$ and $\left.V_{\mathrm{H}}\right)$. Polarized scattering is caused by two contributions: one originating from concentration or density fluctuations - that is, fluctuations of the isotropic part of polarizability - and the other originating from fluctuations of the anisotropy of the polarizability and orientation of the optical axis. Unlike polarized scattering, the depolarized scattering depends only on the fluctuations of optical anisotropy and orientation.

In our experiments we used two polarization modes: $V_{\mathrm{V}}$ and $H_{\mathrm{V}}$. For the system under investigation, the $V_{\mathrm{H}}$ mode could have been used instead of the $H_{\mathrm{V}}$ mode, since depolarized intensities are equal if the sample does not show a strong optical rotation (which is true for the samples studied in this work). However, the direction of shear flow with respect to the polarizer is also important. Using the $H_{\mathrm{H}}$ mode instead of the $V_{\mathrm{V}}$ mode for an optically anisotropic system would decrease the polarized intensity, since the shear flow is in the V-direction and molecules orient preferentially in the flow-gradient plane.

In the Stein-Wilson treatment, a special case of random orientation correlations is considered in which it is assumed that the probability of correlations in orientation of the optic axes of two volume elements depends only upon their separation and not upon the angle that the optic axes make with the vector interconnecting them. This assumption leads to the development of spherically symmetric domains whose scattering is described by: ${ }^{[32]}$

$$
\begin{aligned}
& I_{H_{\mathrm{V}}}(q) \sim\left\langle\delta^{2}\right\rangle \int_{0}^{\infty} f(r) \frac{\sin (q r)}{q r} 4 \pi r^{2} \mathrm{~d} r \\
& I_{V_{\mathrm{V}}}(q)-\frac{4}{3} I_{H_{\mathrm{V}}}(q) \sim\left\langle\eta^{2}\right\rangle \int_{0}^{\infty} \gamma(r) \frac{\sin (q r)}{q r} 4 \pi r^{2} \mathrm{~d} r
\end{aligned}
$$

where $\left\langle\delta^{2}\right\rangle$ is the mean-square anisotropy, $f(r)$ is the correlation function of these orientation fluctuations, $\left\langle\eta^{2}\right\rangle$ is the meansquare density fluctuation and $\gamma(r)$ its correlation function. The magnitude of the scattering vector is $|q|=(4 \pi / \lambda) \sin (\theta / 2)$, with $\theta$ being the polar scattering angle and $\frac{\sin (q r)}{q r}$ is the form factor for spherically symmetric systems. As no a priori information is available on the nature of the correlation functions $f(r)$ and $\gamma(r)$, it is more convenient to describe the evolution of the scattering patterns in terms of the density invariant $Q_{\eta}$ and the orientation invariant $Q_{\delta}$ that are defined as integrated scattering intensities: ${ }^{[33,34]}$

$$
\begin{aligned}
& \underline{Q}_{\eta}=\int_{0}^{\infty}\left(I_{V_{\mathrm{V}}}-(4 / 3) I_{H_{\mathrm{V}}}\right) q^{2} \mathrm{~d} q \sim\left\langle\eta^{2}\right\rangle \\
& \underline{Q}_{\delta}=\int_{0}^{\infty} I_{H_{\mathrm{V}}} q^{2} \mathrm{~d} q \sim\left\langle\delta^{2}\right\rangle
\end{aligned}
$$

These invariants are independent of the form of the correlation function (which characterizes the geometry of the scattering system) and allow values of the mean-square density fluctuations, as well as mean-square anisotropy, to be calculated. However, it should be noted that in practice, instead of using Equation (3) and (4), the intensities are integrated within a finite $q$ range. Also, as mentioned in the introduction, a two-dimensional integration over both azimuthal and polar scattering angles is required for interpretation of the invariant in the case of non-isotropic scattering patterns.

For a random assembly of spherulites in an isotropic matrix, both invariants can also be expressed in terms of structural, optical, and crystalline parameters of the growing spherulites: ${ }^{[33,34]}$

$$
\begin{aligned}
& \underline{Q}_{\eta} \sim\left\langle\eta^{2}\right\rangle=\phi_{\mathrm{s}}\left(1-\phi_{\mathrm{s}}\right)\left(\bar{\alpha}_{\mathrm{s}}-\alpha_{0}\right)^{2} \\
& \underline{Q}_{\delta} \sim\left\langle\delta^{2}\right\rangle=\phi_{\mathrm{s}} \delta_{\mathrm{s}}^{2}
\end{aligned}
$$

with $\bar{\alpha}_{\mathrm{s}}$ the average polarizability of a spherulite:

$$
\bar{\alpha}_{\mathrm{s}}=\phi_{\mathrm{cr}, \mathrm{s}} \alpha_{\mathrm{cr}}+\left(1-\phi_{\mathrm{cr}, \mathrm{s}}\right) \alpha_{\mathrm{am}}
$$

and $\delta_{s}$ the spherulite anisotropy:

$$
\delta_{\mathrm{s}}=\phi_{\mathrm{cr}, \mathrm{s}} \delta_{\mathrm{cr}}^{0} f_{\mathrm{cr}, \mathrm{s}}+\left(1-\phi_{\mathrm{cr}, \mathrm{s}}\right) \delta_{\mathrm{am}}^{0} f_{\mathrm{am}, \mathrm{s}}+\delta_{\mathrm{F}} \approx \phi_{\mathrm{cr}, \mathrm{s}} \delta_{\mathrm{cr}}^{0} f_{\mathrm{cr}, \mathrm{s}}
$$

Here $\phi_{\mathrm{s}}$ is the volume fraction of spherulites, $\alpha_{\mathrm{cr}}$ and $\alpha_{\mathrm{am}}$ are the average polarizabilities of the crystalline and amorphous regions (of which a spherulite consists), $\alpha_{0}$ is the polarizability of the surrounding melt and $\phi_{\mathrm{cr}, \mathrm{s}}$ is the crystalline volume fraction within a spherulite. $f_{\mathrm{cr}, \mathrm{s}}$ and $f_{\mathrm{am}, \mathrm{s}}$ are Hermans-type orientation functions describing the orientation of crystalline and amorphous zones with respect to the radius of the spherulite, $\delta_{\mathrm{cr}}^{0}=\left(\alpha_{\mathrm{r}}-\alpha_{\mathrm{t}}\right)_{\mathrm{cr}}$ and $\delta_{\mathrm{am}}^{0}=\left(\alpha_{\mathrm{r}}-\alpha_{\mathrm{t}}\right)_{\mathrm{am}}$ are the values of the intrinsic anisotropies of the pure crystal and the amorphous segments and $\delta_{\mathrm{F}}$ is the form anisotropy of the crystals within the spherulite. While this last term is usually neglected, it could be significant (e.g., in the presence of elongated structures). Assuming that $f_{\mathrm{cr}, \mathrm{s}} \gg f_{\mathrm{am}, \mathrm{s}}$, $\delta_{\mathrm{f}} \approx 0$, and $\delta_{\text {am }}^{0} \approx 0$, the right hand side of Equation (8) is obtained.

It is clear that Equation (5-8) make the SALS invariants powerful tools for monitoring structure development during meltphase separation, crystallization, and melting of complex systems. Moreover, to evaluate the length scales at which these phenomena occur, the time evolution of the scattering intensities is often analyzed by the Debye-Bueche model. ${ }^{[59]}$ This model requires a biphasic system where the refractive indices of the two 
phases are close. Therefore, when only isotropic elements are present in the material during crystallization, this assumption can be considered as fulfilled. However, once the anisotropic elements appear, this assumption is no longer valid, since a relatively large contrast in refractive index is then present. The Debye-Bueche analysis has been developed in the case of circularly symmetric scattering. In this case, the angular dependence of the scattering intensities can be described by the Debye-Bueche type scattering function: ${ }^{[59]}$

$$
I(q)=\left(\frac{A}{1+\xi^{2} q^{2}}\right)^{2}
$$

with $A$ a constant. As a result, the Debye-Bueche type plots $\left(I(q)^{-\frac{1}{2}}\right.$ versus $\left.q^{2}\right)$ should show a linear dependence:

$$
I(q)^{-1 / 2}=\frac{1}{A}+\frac{\xi^{2}}{A} q^{2}
$$

The correlation length, $\xi$, is then found as the square root of the ratio of the slope to the intercept.

\section{Results and Discussion}

\section{Typical Curves and Patterns}

As mentioned in our previous studies of $\mathrm{iPB}^{[57,58]}$ the nucleation density of the samples under study is very low, which makes it very difficult to use SALS for the study of crystallization under quiescent and very mild shear conditions. The area covered by the laser beam is, compared to the size of and the distance between the growing spherulites, too small to give a representative result for the global crystallization process. Under these conditions, the obtained scattering patterns are often distorted, and the use of invariants is therefore meaningless. In order to overcome this difficulty, all results presented in this paper were obtained using sufficiently high shearing conditions, since this drastically enhances the nucleation density.

For most semi-crystalline polymers, experimental SALS patterns deviate substantially from ideal patterns because of several morphological features of real spherulites. ${ }^{[60,61]}$ These include the internal structure of the spherulite, distribution of spherulite sizes, impingement and incomplete growth. However, it is generally concluded that the difference between the experimental and predicted SALS pattern can, to a large extent, be direction. attributed to the internal structure of the spherulite and particularly to the spherulite internal disorder. ${ }^{[61]}$ The effect of internal disorder - that is, fluctuations in the orientation of the optic axis of the crystals within the spherulites - on $H_{\mathrm{V}}$ scattering patterns decreases the intensity maximum at an azimuthal angle of $45^{\circ}$ and increases the scattering intensity at all other angles. This causes the expected four-leaf pattern in the $H_{\mathrm{V}}$ scattering to be less pronounced or even indistinguishable, which is also the case for the studied iPB sample. The scattering patterns only display a characteristic four-leaf pattern at high shear rates and/or low crystallization temperatures (see Figure 2). Under these conditions, the more perfect internal structure of the spherulites and a more uniform distribution of spherulite sizes reduce the deviation from the ideal pattern.

For flow parallel to the polarization direction, strong streaks develop at the early stages of crystallization, as can be seen in Figure 2. In $V_{\mathrm{V}}$ mode, streaks are more pronounced than in $H_{\mathrm{V}}$ mode due to the higher scattering intensity. The fact that the SALS streaks orient perpendicular to the flow direction indicates that (due to the Fourier transform procedure) actual scatterers are oriented along the flow direction or at least in the flow-gradient plane. This preferential orientation of the scatterers along the flow direction is the consequence of elongated structures (threads) formed by the flow. At later times, scattering patterns show a tendency to circular symmetry. This is the result of growing morphologies that are randomizing in space, indicative of spherulitic overgrowth. However, the weakly elongated scattering patterns in Figure 2 indicate that these spherulites may be deformed into ellipsoids. According to Fukushima et al. ${ }^{[45-47]}$ and Zhang et al., ${ }^{[48]}$

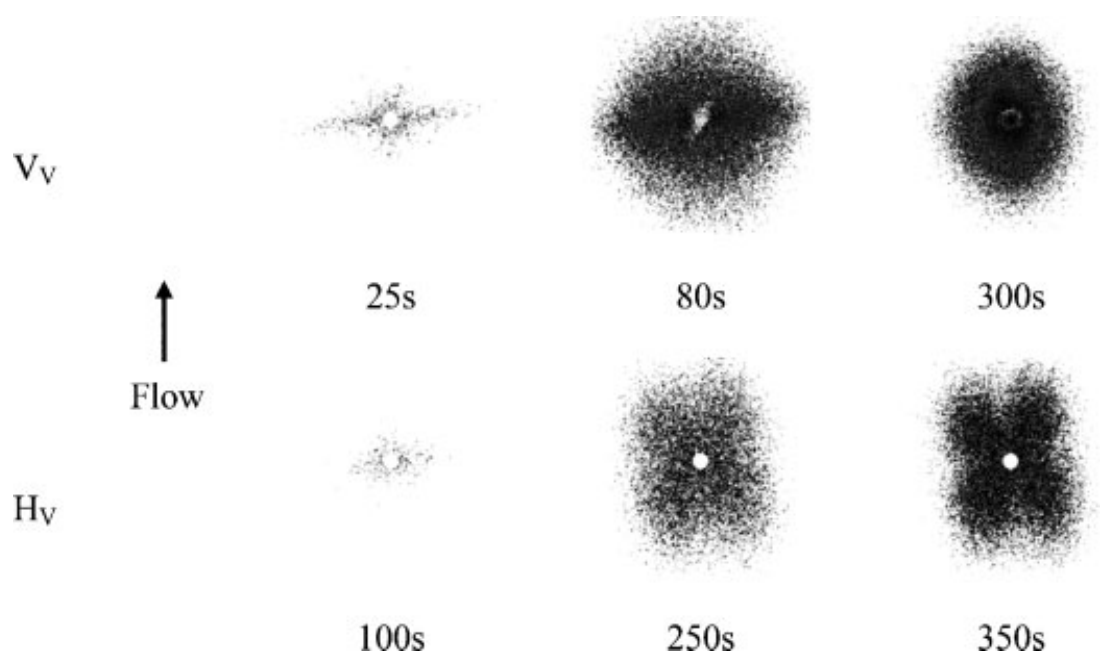

Figure 2. $V_{\mathrm{v}}$ and $H_{\mathrm{V}}$ images during shear-induced crystallization of PBo400 at $98^{\circ} \mathrm{C}$. Shearing conditions: $\dot{\gamma}=100 \mathrm{~s}^{-1}$ and $\gamma=100$. The flow direction is parallel to the polarization 
this anisotropic streak-like scattering is an indication of shish-like structures, and the disappearance of anisotropy at the late stage of crystallization is ascribed to the shish-kebab-like structures impinging into each other and the kebabs growing and penetrating into spaces of other shish-kebab-like structures. However, since these shishlike structures are already observed at very low shear rates (below the shear rate for coil-stretch transition) and since the streaks disappear after complete crystallization, the observed structures must be different from those usually referred to as shish-kebabs. The anisotropic scattering can also indicate the presence of some oriented domains remaining after shear, which do not lead to shish-kebab formation.

Figure 3 shows the time variation of the invariants $\underline{Q}_{\eta}$ and $Q_{\delta}$ for PB0400 crystallized at $98^{\circ} \mathrm{C}$ after a shear pulse with $\dot{\gamma}=10 \mathrm{~s}^{-1}$ and $\gamma=100$. (Integration according to Equation (3) and (4) over all azimuthal angles.) $Q_{\eta}$ and $Q_{\delta}$ are measures for the growing fluctuations of density and orientation, respectively, and in Figure 3 they display the typical behavior for polymer crystallization. This behavior was observed for all the experimental conditions investigated. Following an induction period, the density fluctuations $\left\langle\eta^{2}\right\rangle$ start to increase at the early stages, pass through a maximum and decrease at later stages of crystallization when the crystalline phase becomes dominant. From Equation (5), the maximum in $\left\langle\eta^{2}\right\rangle$ should occur when $\phi_{s}=0.5$ (assuming the average polarizability of the spherulite does not change); that is, the spherulites grow and occupy $50 \%$ of the volume. Here the time for this maximum is defined as $t_{\underline{Q}_{\eta}}$. Next, $\underline{Q}_{\eta}$ decreases and reaches zero [see Equation (5)] because the clusters become volume

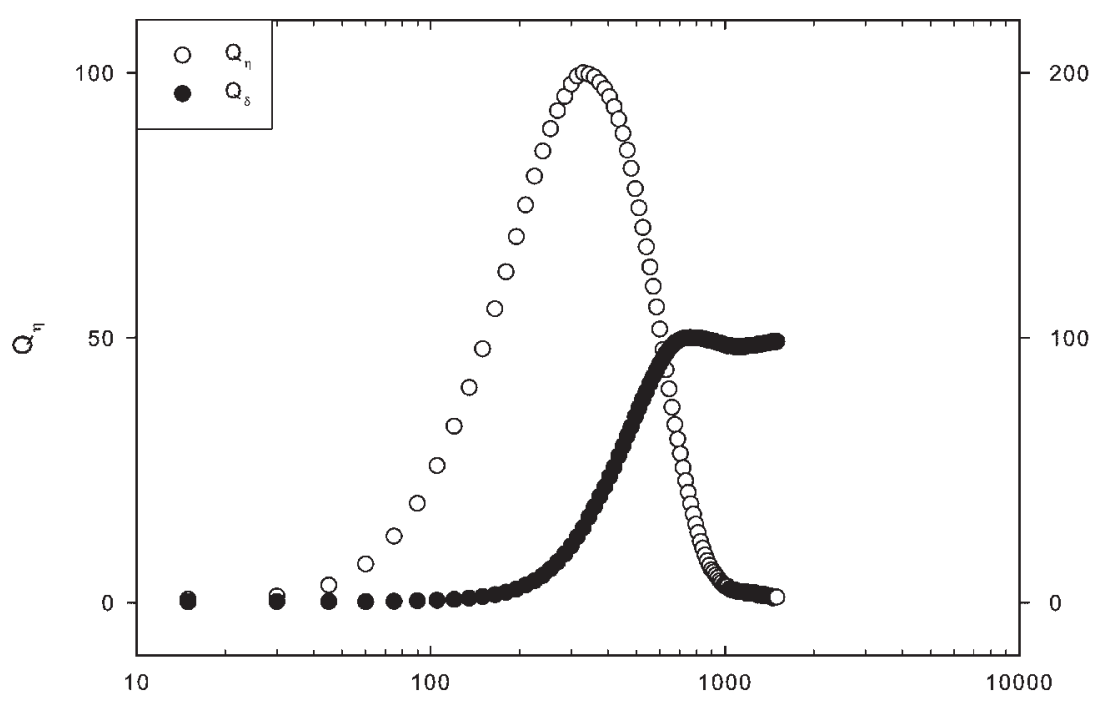

$t(s)$

Figure 3. Evolution of invariants $\underline{O}_{\eta}$ and $\underline{O}_{\delta}$ during crystallization of PBo40o at $98^{\circ} \mathrm{C}$. Shearing conditions: $\dot{\gamma}=10 \mathrm{~s}^{-1}$ and $\gamma=100$. filling $\left(\phi_{s} \approx 1\right)$ and the average polarizability of the surrounding matrix, which now consists of similar spherulites, becomes close to that of the spherulites $\left(\alpha_{0} \approx \bar{\alpha}_{\mathrm{s}}\right)$. Negative values of $\underline{Q}_{\eta}$, sometimes observed at longer times, occur for conditions where $I_{H_{\mathrm{V}}}(q) \sim I_{V_{\mathrm{V}}}(q)$. The negative values indicate a breakdown of the approach of Yoon et al. ${ }^{[33]}$ and Koberstein et al., ${ }^{[34]}$ possibly due to the onset of multiple scattering. Although included for completeness, this does not affect the discussion of the early stages of crystallization. The orientation fluctuations $\left\langle\delta^{2}\right\rangle$, due to the optical anisotropy of crystals, develop at much later stages, when density fluctuations already reached a significant level, and show sigmoidal growth. The time variation of $\left\langle\delta^{2}\right\rangle$ is conceivable in light of Equation (6) and (8): during crystallization it should increase linearly with $\phi_{\mathrm{s}}$ and as the square of $\phi_{\mathrm{cr}, \mathrm{s}}$. After the clusters have become volume filling, any increase in both invariants is a further indication of an increase in the fraction of crystals within the spherulites, $\phi_{\mathrm{cr}, \mathrm{s}}$, or their degree of perfection or orientation. Thus, from Equation $(5-8)$ it can be concluded that, in the case of spherulitic polymer crystallization, the density invariant $\underline{Q}_{\eta}$ is the result of the external contrast - that is, the difference in polarizability between matrix and spherulites - whereas the orientation invariant $Q_{\delta}$ depends on the internal contrast - that is, the anisotropy of the crystalline and amorphous parts within the spherulite.

The difference in the onset time of $\underline{Q}_{\eta}$ and $Q_{\delta}$, observed in Figure 3, has been reported previously by Okada et al., ${ }^{[35]}$ Pogodina et al. ${ }^{[37,38]}$ (both for quiescent and shear-induced crystallization), Elmoumni et al. ${ }^{[39]}$ (both for quiescent and shear-induced crystallization), Somwangthanaroj et al., ${ }^{[49]}$ Devaux et al. ${ }^{[40,41]}$ (both for quiescent and shear-induced crystallization) and Imai et al., ${ }^{[36]}$ but the reason for this has rarely been addressed. We believe that this difference in onset time is polymer specific and depends on the crystallization temperature, the shearing conditions and the sensitivity of the camera used for the scattering experiments. ${ }^{\circ}$ This explains why there have been observations where the maximum in $Q_{\eta}$ occurs when $Q_{\delta}$ is still low, ${ }^{[37-41]}$ when $Q_{\delta}$ has reached about half of its final value, ${ }^{[35,36]}$ or when $Q_{\delta}$ is already close to its maximum. ${ }^{[38]}$

Okada et al. ${ }^{[35]}$ were one of the first to point out the unexpected difference in onset time between the density and orientation fluctuations for the quiescent crystallization of an iPP. It was ascribed to the development of an isotropic embryo as the precursor of a 
spherulite. The authors mentioned two possibilities for this isotropic embryo: a high-density domain by thermal fluctuations, or a less-ordered crystalline domain. According to them, if the former was the case, $\left\langle\eta^{2}\right\rangle$ and $\left\langle\delta^{2}\right\rangle$ would show an abrupt increase when the high-density domain transforms to the crystalline domain, which is indeed not the case. Therefore they concluded that the latter possibility was more realistic. However, in our opinion, this transformation from a high-density to a crystalline domain could also occur gradually without necessarily causing an abrupt increase in the invariants. To validate their assumption of the presence of an isotropic embryo, the authors also showed that, in the early stages of crystallization, the degree of disorder in the fibrillar arrangement within a spherulite is very large. In the same publication they also managed to interpret the temperature dependence of the growth of the isotropic embryo (represented by $Q_{\eta}$ ) by the Hoffman-Lauritzen theory, again suggesting its crystalline nature. Pogodina et al. ${ }^{[37]}$ explained the observed difference in onset time in essentially the same way. The upturn in $\underline{-}_{\eta}$ at the early stages is caused by primary crystallization during which clusters or fibrous crystalline strands grow radially and form an interconnected network of fibrous crystals (gelation). These crystals are themselves anisotropic, but since their fraction is low, the anisotropy of the cluster is also low. The fact that $\underline{Q}_{\eta}$ reaches a maximum has to do with the fact that these clusters form a volume spanning network. The strong increase in $Q_{\delta}$ after the maximum in $Q_{\eta}$ suggests the presence of secondary crystallization: a process of internal crystallization and ordering which proceeds within the impinged clusters. A bit surprisingly, Akpalu et al. ${ }^{[51]}$ stated that, for the crystallization of a homogeneous copolymer, the onset of changes in the $H_{\mathrm{V}}$ and $V_{\mathrm{V}}$ invariant (which differs from the density invariant $Q_{\eta}$ ) occurs simultaneously, although one of the figures clearly shows a faster upturn in the $V_{\mathrm{V}}$ invariant. Nevertheless, based on $\mathrm{X}$-ray measurements, they also came to the conclusion that a significant fraction of the crystallinity develops after the spherulites have become volume filling. The above findings all seem to suggest that the observed density fluctuations result from the presence of weakly anisotropic domains, rather than being an indication of true densification before the onset of crystallization. These weakly anisotropic domains are loosely structured spherulites for which the crystalline parts are too tiny to cause a measurable anisotropy by themselves. With ongoing crystallization, the internal structure of these spherulites matures, and orientation fluctuations caused by the growing anisotropic entities also start to play a role.

\section{The Effect of Shear}

The invariants used in the previous section (Figure 3) were calculated for a shear-induced crystallization experiment, because the low nucleation density of iPB does not allow us to measure under quiescent conditions. However, is the Stein-Wilson approach still valid to analyze these shearinduced data? As long as the scattering is isotropic the same method of analysis for both the experiments under flow and the quiescent experiments can be justified. For example, in their shear-induced crystallization experiments Devaux et al. ${ }^{[0,41]}$ showed that the level of anisotropy was of the same order as that for static experiments by measuring the anisotropy of the scattering patterns in the $V_{\mathrm{V}}$ mode by the second order moments of the intensity. On the other hand, when the scattering becomes anisotropic the assumptions of the Stein-Wilson analysis are no longer fulfilled and two-dimensional invariants have to be used. This is the approach that was used here and that was also applied by the group of Winter, ${ }^{[38,39]}$ which was the first to combine SALS with shear-induced crystallization experiments.

Next, the effect of shearing on the evolution of the SALS patterns was investigated systematically. In Figure 4 calculated values of $Q_{\eta}$ and $Q_{\delta}$ for PB0400 at $98^{\circ} \mathrm{C}$ for different shear rates and a constant strain are plotted as a function of the crystallization time.

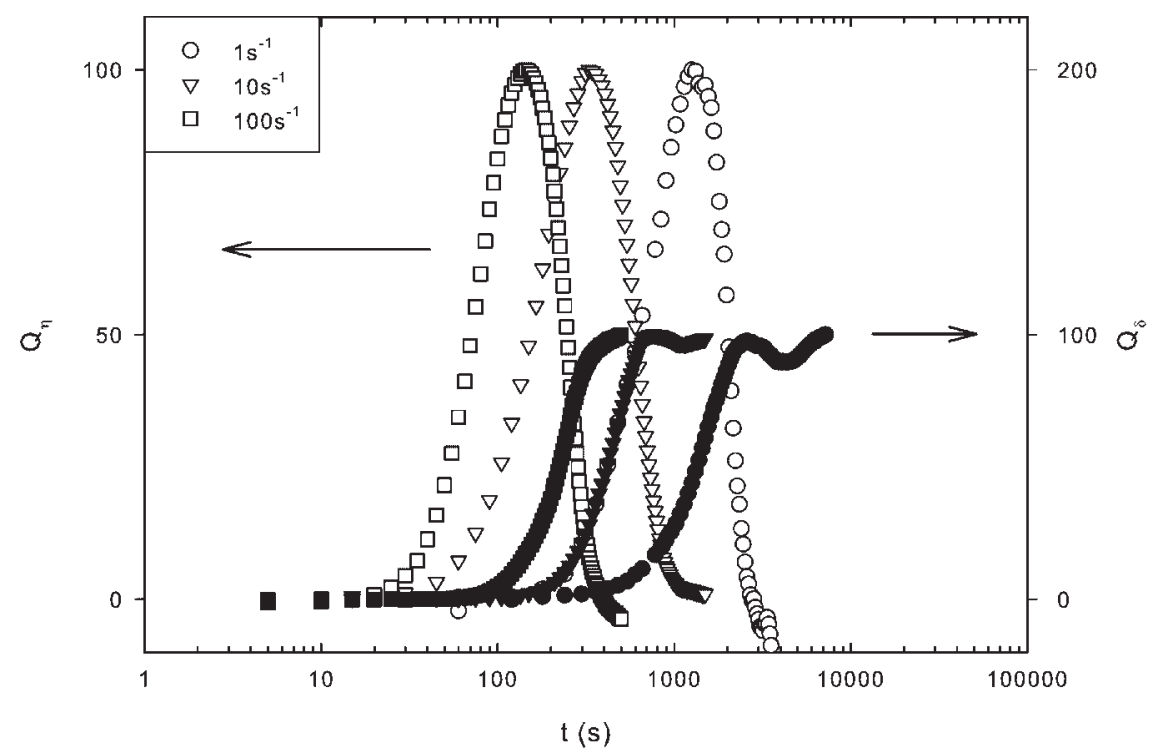

Figure 4. Time variation of $\underline{Q}_{\eta}$ (open symbols) and $\underline{Q}_{\delta}$ (filled symbols) invariants during crystallization of PBO400 at $98{ }^{\circ} \mathrm{C}$ for various shear rates and constant $\gamma=100$. 


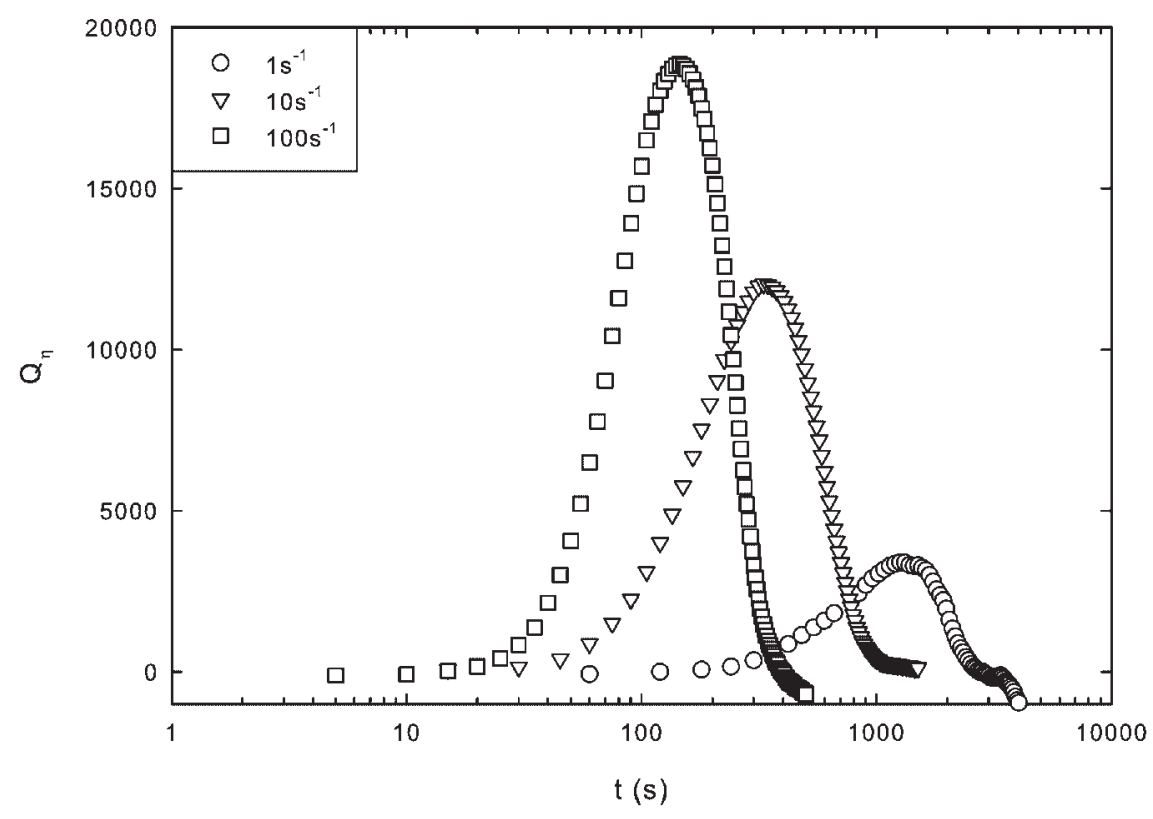

Figure 5. Effect of shear rate on the evolution of the scattering invariant $Q_{\eta}$ at $98{ }^{\circ} \mathrm{C}$ for various shear rates and constant $\gamma=100$.

Over the entire range of shear rates explored, density fluctuations appear at the very early times, pass through a maximum, and decrease at later stages. The orientation fluctuations always develop at much later stages and show sigmoidal growth. For both curves the maximum is reached earlier as more shear (higher shear rate at constant strain or higher strain at constant shear rate) is applied to the samples. The fact that the orientation fluctuations occur earlier when the polymer is subjected to flow is coherent with turbidity results ${ }^{[57]}$ and does not contain extra information. On the contrary, the similar observations for $\underline{Q}_{\eta}$ suggest that the density fluctuations are also coupled with the flow. Figure 5 shows the evolution of the scattering invariant $Q_{\eta}$ for a range of shear rates. Increasing the shear rate affects both the relative importance of the density fluctuations and the kinetics of their development and disappearance: as the shear rate is increased, the maximum in $Q_{\eta}$ shifts to shorter times and higher values. According to Equation (5), the increase in the maximum value of the density invariant $Q_{\eta}$ with shear rate can be ascribed to an increase the average polarizability of the spherulites, $\bar{\alpha}_{s}$.

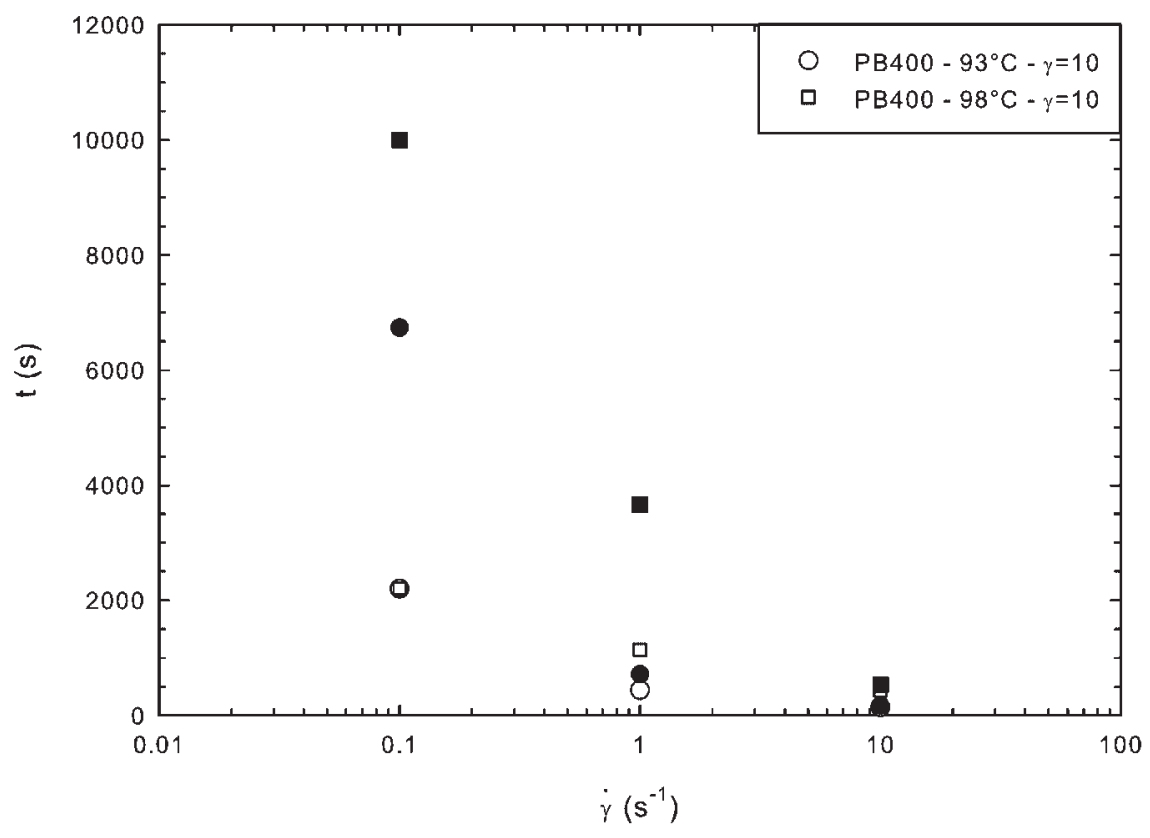

Figure 6. $t_{Q_{\eta}}$ (open symbols) and $t_{Q_{\delta}}$ (filled symbols) for the crystallization of PBo40o under various conditions.
The above observations are in agreement with results of Elmoumni et al. ${ }^{[39]}$ and Devaux et al.${ }^{[0,41]}$ Moreover, the latter authors found that the effects of mild flow conditions on the evolution of the SALS invariants were similar to the effect of temperature on quiescent crystallization. Although it is not clear whether or not the data of Pogodina et al. ${ }^{[37]}$ represent absolute values, they show the same trend: an increase in the relative importance and the kinetics of both invariants with decreasing temperature. In our study, temperature did not have a significant effect on the absolute value of the invariants, most likely because the applied shear flow obscures the effect of temperature. Devaux ${ }^{[41]}$ also found that, after applying shear flow, the decrease in the characteristic time with respect to static conditions was the same for both $t_{O_{\eta}}$, the time corresponding with the maximum in the density fluctuations, and $t_{Q_{\delta}}$, the time to reach $50 \%$ of the orientation fluctuations. According to the author, this indicates that the effect of flow on the orientation fluctuations is directly related to the effects on the density fluctuations observed in the early stages of crystallization. As can be seen from Figure 6, this statement is probably not general and 
depends on the polymer characteristics. In any case, it is not the case for our experiments: the time between $t_{Q_{\eta}}$ and $t_{Q_{\delta}}$ decreases with increasing shear rate, indicating that the spherulites crystallize faster with increasing shear rate. Within the range of shear rates and shear strains investigated, we did however not reach the situation where $t_{Q_{\delta}} \ll t_{Q_{\eta}}$, as was the case for the shear-induced experiment of Pogodina et al. ${ }^{[38]}$ These authors found that orientation fluctuations had already reached saturation at about the time when density fluctuations passed through a maximum, indicating that the system of solidifying clusters (threads) rapidly reaches the state of the final crystallinity due to orientational and deformational effects of shear flow.

\section{SALS Compared to Other Techniques}

In order to elucidate the typical behavior of the scattering invariants and the physical meaning of density fluctuations, many studies have combined SALS measurements with rheological and/or other optical techniques. The evolution of rheological and optical properties follows the crystallization process, starting from the physical melt behavior to the fully crystallized solid state, and this allows probing different regimes of crystallization - for example, nucleation, gelation, and crystallinity growth. As a result, scattering and transmittance data from rheooptical measurements, complemented by microscopy, calorimetry and rheology provide important and diverse information for understanding the structure/property relationship during crystallization. In the past, several attempts have been made to compare the time scales of these observations in order to merge them into a unified framework. In the next paragraph these studies are briefly summarized. The different techniques that have been used in combination with SALS are also listed in Table 2.

Pogodina et al. ${ }^{[37]}$ compared the time scales of crystallinity growth governed by the Avrami time (from DSC), connectivity growth governed by the gel time (from rheology), and evolution of clusters governed by a characteristic timescale for structure development (from SALS) during the early stages of crystallization of iPP. They found that the time for the maximum in the density fluctuations $t_{Q_{\eta}}$ is very close to the gel time $t_{c}$, but much shorter than the corresponding Avrami time $t_{\mathrm{A}}$ - that is, the time by which the relative degree of crystallinity has reached $63 \%$ of its final value. From the former they concluded that the liquid-solid transition occurs very close to the instant of the maximum of density fluctuations in the system. The latter suggests that gelation (i.e., formation of an interconnected network) occurs at the very early stages of the crystallization process, and supports the fact that nearly all of the crystallization occurs on the template of the solid structure of the critical gel. Indeed, by employing DSC, they showed that solidification occurs at a relative degree of crystallinity less than $3 \%$ and the maximum in density fluctuation at relative crystallinity less than $2 \%$. In a later study, ${ }^{[38]}$ the same authors performed SALS, birefringence and turbidity measurements in a single experimental run and combined it with rheological measurements and optical microscopy. For quiescent crystallization it was observed that the SALS orientation invariant $Q_{\delta}$ grows at the same rate as the storage modulus $G^{\prime}$, suggesting that solidification parallels the development of mean-square optical anisotropy during crystallization. Again the degree of crystallinity at the gel point and the maximum of density fluctuations were calculated. However, the values obtained were about one order of magnitude larger than those presented in their previous study. This discrepancy may be caused by the different methods used for the determination of the crystallinity, extrapolation of the DSC data, and real differences in the crystallization process (e.g., due to surface effects). After comparing rheological, scattering and microscopical data, it was clear that structure development can be observed microscopically before the gel point is reached and that the maximum in density fluctuations occurs close to the instant of spherulitic impingement. This suggests that the higher degree of crystallinity calculated in their second study ${ }^{[38]}$ is more realistic. They also speculated that the same trend would be true for the case of shear-induced crystallization - that is, the instant of the maximum of density fluctuation occurs in the vicinity of the gel point. Elmoumni et al. ${ }^{[39]}$ tried to correlate the molecular time scales with the morphological structures that arise upon application of shear at the onset of iPP isothermal crystallization. From their data, it can again be seen that spherulites are already visible before the maximum in density fluctuations, even halfway through this time period, and that the maximum in density fluctuations occurs before the moment of spherulitic impingement. This is in agreement with the observations made by Pogodina et al. ${ }^{[38]}$ Devaux et al..$^{[40,41]}$ showed that the evolution of the overall crystallization kinetics, monitored by direct transmission of depolarized light, is different from the evolution of $Q_{\delta}$ : the fluctuations in anisotropy are more sensitive to the morphology development. This also implies that density fluctuations are detected before the increase in the degree of crystallization, measured by depolarized light intensity. However, optical microscopy showed that spherulites can already be seen before there was an upturn in the degree of crystallinity, caused by the lower detection limit of the transmission technique. The above considerations confirm the need of comparing density fluctuations with direct observations of the developing morphology. The group of Kaji and Imai (e.g., ref. ${ }^{[21]}$ ) observed that, for cold 
crystallization of PET, sPS and iPS, the invariant $Q_{\delta}$ increases exponentially with annealing time, levels off somewhat and then again increases exponentially, due to the formation of the spherulite texture. This behavior of $Q_{\delta}$, a characteristic feature of spinodal decomposition, was observed in the induction period of crystallization, before crystallinity was detected with DSC, WAXS and density measurements, and it suggests that the dense domains in the induction period are not the embryos predicted by classical nucleation theory. Somwangthanaroj et al. ${ }^{[49]}$ used POM to qualitatively confirm features quantified by SALS. For an intercalated poly(propylene) nanocomposite there already are some spherulites visible when the maximum in $\underline{Q}_{\eta}$ is reached, but they are still far from impingement. For pure iPP the images are less clear, but it seems that spherulites are close to impingement when the maximum in $\underline{Q}_{\eta}$ is reached. This clearly shows that nanoparticles have an effect on the early stages of polymer crystallization. Akpalu et al. ${ }^{[51]}$ found that, during copolymer crystallization, the percent transmission goes through a minimum when the $V_{\mathrm{V}}$ invariant reaches its maximum. Shortly after, both the spherulite radius (obtained from $H_{\mathrm{V}}$ scattering) and the transmission attain a limiting value. Nishida et al. ${ }^{[54]}$ combined SALS with optical microscopy in order to characterize the structure variation preceding the crystallization of PET after rapid quenching. They observed the emergence of fluctuations with quasi-periodicity, a characteristic of spinodal decomposition. Wang et al. ${ }^{[55]}$ extended this work to cold crystallization of sPS. Again, interconnected domains imbedded within the well-defined spherulites were observed in all the crystallized samples under phase contrast microscopy, suggesting that spinodal phase separation takes place in sPS when heated from the glassy state. Owing to a small difference in the refractive index between sPS dense and sPS less dense domains, light scattering was found to be not as effective as optical microscopy in detecting this phase-separated morphology. For quiescent crystallization, Fukushima et al. ${ }^{[46]}$ already observed depolarized light scattering before crystallization was detected with DSC. In a later study, ${ }^{[47]}$ they also reported that, again for quiescent crystallization, the length of the induction period for depolarized light scattering was almost identical to that for the appearance of spherulites in POM measurements, implying that their DSC measurements have a limited sensitivity.

In the present study the SALS measurements were also complemented with observations from POM. Figure 7 shows, in combination with the SALS invariants $Q_{\eta}$ and $Q_{\delta}$, optical micrographs at different times during the

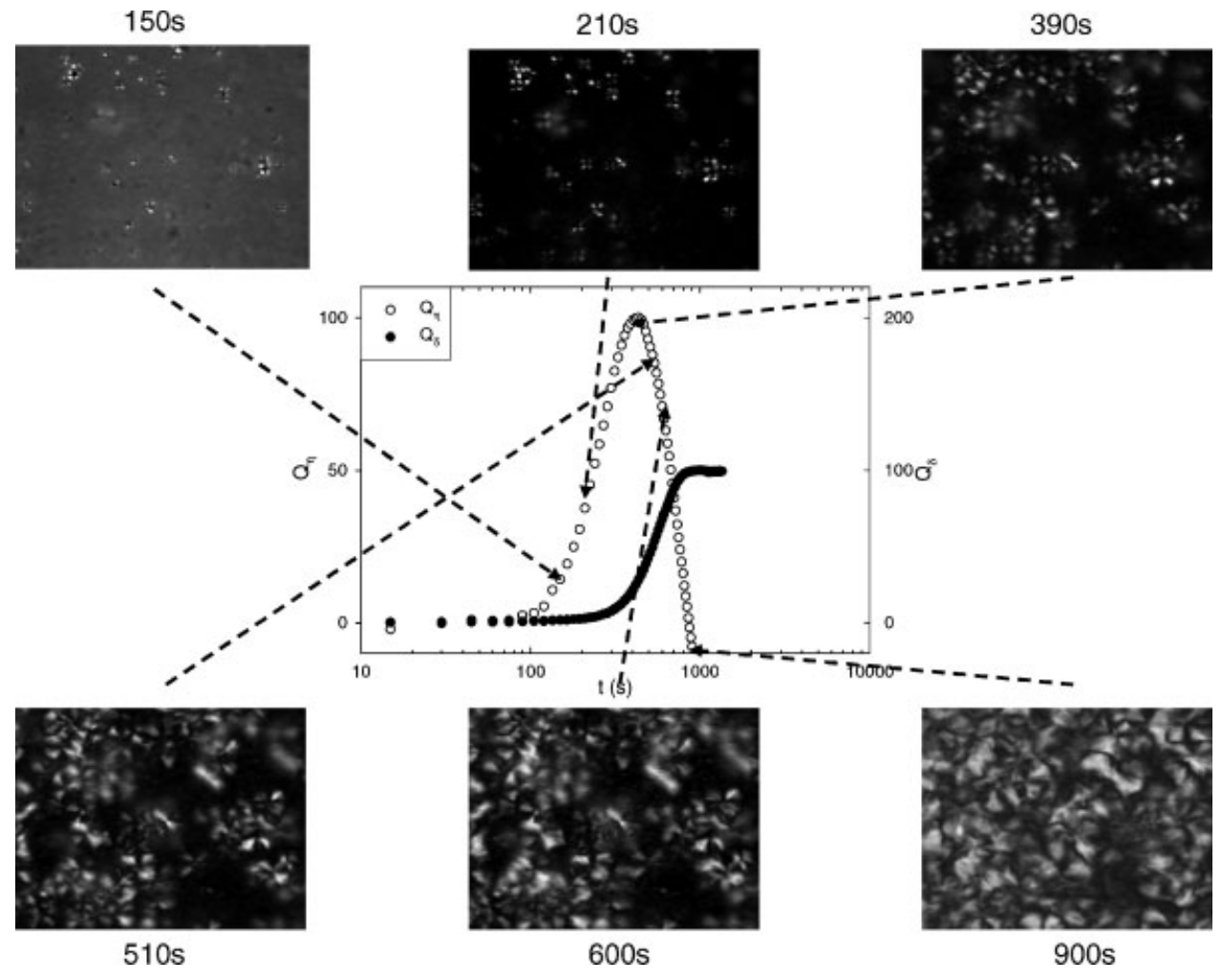

Figure 7. SALS invariants and optical micrographs showing the structure evolution during crystallization of PBo400 at $98^{\circ} \mathrm{C}$. Shearing conditions: $\dot{\gamma}=10 \mathrm{~s}^{-1}$ and $\gamma=10$. 


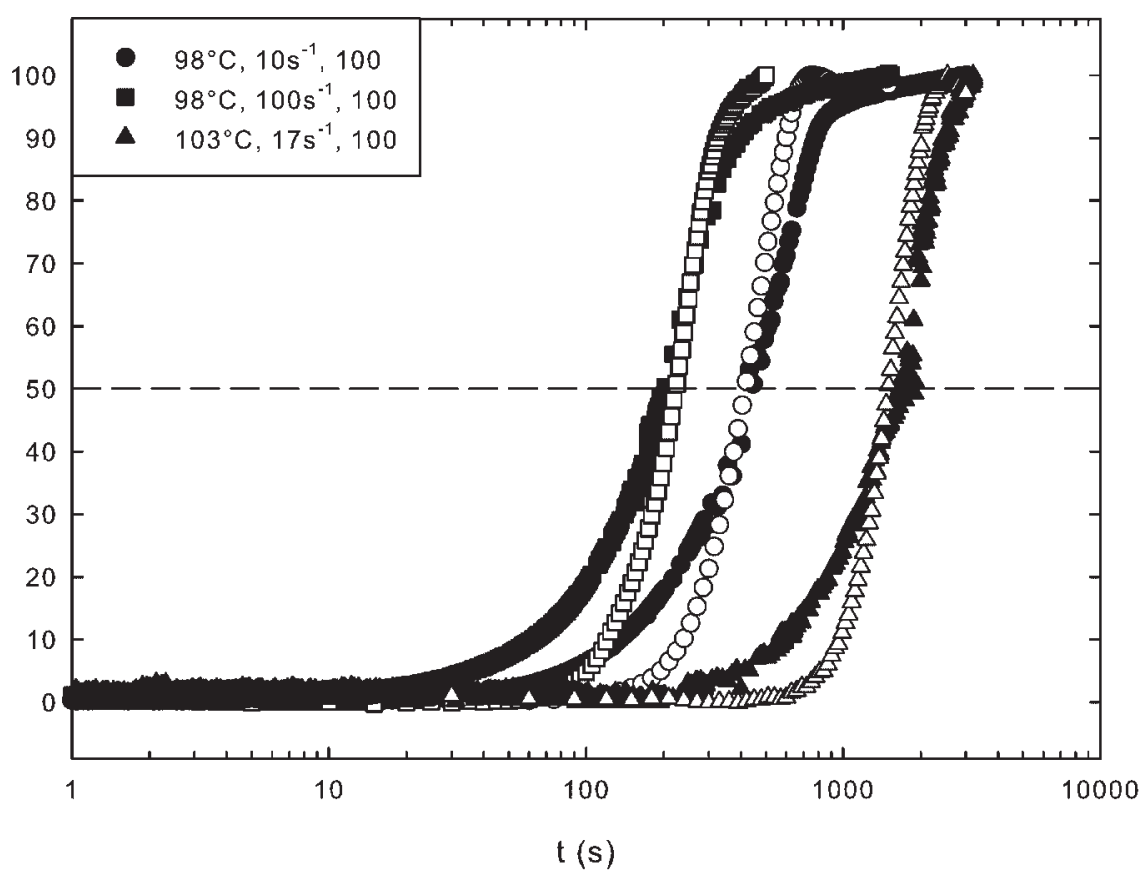

Figure 8. Evolution of birefringence $\Delta n^{\prime}$ (filled symbols) and orientation fluctuations $Q_{\delta}$ (open symbols) during crystallization of PBo40o at various conditions. The dashed horizontal line is added for clarity.

crystallization of PB0400 at $98^{\circ} \mathrm{C}$. Structure development can be observed microscopically already during the increase of density fluctuations and the maximum in $\underline{Q}_{\eta}$ clearly occurs before the instant of spherulitic impingement, which is in agreement with the observations made by Pogodina et al. ${ }^{[38]}$ and Elmoumni et al. ${ }^{[39]}$ From Figure 7, it can be seen that the upturn in density fluctuations corresponds with the appearance of small spherulites in the optical micrographs, again suggesting that density fluctuations indicate the presence of weakly anisotropic domains, rather than a densification step preceding nucleation. This is also what one would expect based on Equation (5), which clearly implies that density fluctuations can only be detected after the volume fraction of spherulites has become non-zero; that is, after nucleation. In his doctoral work, Devaux ${ }^{[41]}$ compared, for different crystallization temperatures, the appearance of spherulites with the onset of density fluctuations. He showed that, for low temperatures, both times are very close to each other, as predicted by Equation (5). For flow-induced crystallization Devaux ${ }^{[41]}$ even suggested (however, based on a single experiment) that nuclei are activated before density fluctuations are detected. These results confirm that density fluctuations, at least at the instant we detect them with SALS, do not necessarily develop before the crystalline units.

Another interesting feature of our SALS data is that the evolution of the $Q_{\delta}$ invariant shows very good agreement with the evolution of birefringence $\Delta n^{\prime}$ as measured in a previous study. ${ }^{[58]}$ Especially the half-time of both orientation fluctuations, $t_{Q_{\delta}}$, and birefringence, $t_{0.5}$, seem to correspond extremely well. On the other hand, the birefringence is more sensitive to the development of anisotropy in the beginning of the crystallization, whereas in the late stages $Q_{\delta}$ shows a more pronounced change. This can be seen from Figure 8, where the evolution of both quantities during crystallization is plotted.

What could be the reason for this observed similarity? It is clear that for crystallizing polymers, as studied here, both experimental techniques give qualitative information on the time scale of the ordering process. According to Yoon and Stein ${ }^{[62]}$ the spherulite birefringence $\Delta_{\mathrm{s}}$ may be given by:

$$
\Delta_{\mathrm{s}}=\phi_{\mathrm{cr}, \mathrm{s}} \Delta_{\mathrm{cr}}^{0} f_{\mathrm{cr}, \mathrm{s}}+\left(1-\phi_{\mathrm{cr}, \mathrm{s}}\right) \Delta_{\mathrm{am}}^{0} f_{\mathrm{am}, \mathrm{s}}+\Delta_{\mathrm{F}}
$$

where $\Delta_{\mathrm{cr}}^{0}$ is the intrinsic birefringence of the crystals, $\Delta_{\mathrm{am}}^{0}$ is the intrinsic birefringence of the segments of the amorphous phase, and $\Delta_{\mathrm{F}}$ is the form birefringence of the crystals within the spherulite. The latter two terms in Equation (11) are usually neglected. This expression for the spherulite birefringence [Equation (11)] is very similar to that for the spherulite anisotropy [Equation (8)]. In fact, the Lorentz-Lorenz equation ${ }^{[63]}$ allows birefringence and anisotropy to be related, which may explain the good agreement observed in Figure 8. 


\section{Correlation Length}

As was already mentioned in the paragraph on the analysis of SALS data, the time evolution of the scattering intensities is often analyzed by the Debye-Bueche model to evaluate the length scales at which the observed phenomena occur [Equation (9) and (10)]. This method is, in contrast to the study of the invariants, very delicate due to the small scattering intensities in the early stages of crystallization and in many of the reported studies the model has been applied in various ways, often without taking into account its restrictions. Moreover, it is not clear what the exact meaning is of the calculated correlation length and as a consequence it is difficult to link the results with the developing semi-crystalline structures. In the next paragraph we have summarized and compared SALS studies that report on correlation lengths. Not only will this illustrate the difficulties encountered in applying the Debye-Bueche model, it will also help us to put our results on iPB in a broader perspective. A compact overview of these studies can be found in Table 3 .

Okada et al. ${ }^{[35]}$ showed that the angular dependence of both $I_{H_{\mathrm{V}}}$ and $I_{V_{\mathrm{V}}}-\frac{4}{3} I_{H_{\mathrm{V}}}$ was well described by the Debye-Bueche scattering function. Although they did not mention whether or not both intensities result in the same correlation length, this is not expected to be the case. According to the authors, in the early stages of crystallization the average radius of a spherical isotropic embryo is a function of the correlation length, $\xi$, and the volume fraction $\phi_{\mathrm{s}}$ (both obtained from the evolution of $Q_{\eta}$ ). This average radius was found to be in the range 0.5-1 $\mu \mathrm{m}$. According to Pogodina et al., ${ }^{[37]}$ the correlation length $\xi$ defines the size of the crystalline or amorphous phase, depending on the stage in the crystallization process. The Debye-Bueche plots were applied to circularly averaged $V_{\mathrm{V}}$ intensities. The authors showed that, for different crystallization temperatures, $\xi$ first increases (on the order of the size of the amorphous superstructures), attains a maximum of about $1 \mu \mathrm{m}$ (a characteristic length scale associated with the clusters) and then decreases to a constant value of about 0.6-0.7 $\mu \mathrm{m}$ (a measure of the amorphous regions). When $\xi$ reaches its constant value, the amorphous regions stopped decreasing in size and primary crystallization is complete. The time to reach the maximum correlation length was found to be systematically shorter than those corresponding with the maximum in $Q_{\eta}$ and the gel point. Somwangthanaroj et al. ${ }^{[49]}$ applied the Debye-Bueche analysis to $I_{V_{\mathrm{V}}}$ (after subtracting the melt state scattering) for $I_{H_{V}}=0$ and showed that there is a significant difference in the time-dependent growth of the isotropic morphology in the polymer and the nanocomposite. For pure iPP the correlation length grows to a maximum value of about $1 \mu \mathrm{m}$ (cfr. Okada et al. ${ }^{[35]}$ and Pogodina et al. $^{[37]}$ ) within a period slightly less than the maximum in $Q_{\eta}$ and then decreases again. The intercalated iPP nanocomposite showed a more complex behavior:

Table 3. Overview of the use of the Debye-Bueche correlation length in polymer crystallization experiments.

\begin{tabular}{|c|c|c|c|c|c|c|}
\hline \multirow[t]{2}{*}{ Author/Group } & \multirow[t]{2}{*}{ Material } & $T$ range & \multirow[t]{2}{*}{$I$} & \multirow{2}{*}{$\frac{q^{2} \text { range }}{\mu \mathrm{m}^{-2}}$} & $\xi$ & \multirow[t]{2}{*}{ Measure for } \\
\hline & & ${ }^{\circ} \mathrm{C}$ & & & $\mu \mathrm{m}$ & \\
\hline Okada et al. ${ }^{[35]}$ & iPP & $128-143$ & $I_{H_{\mathrm{V}}}$ and $I_{V_{\mathrm{V}}}-4 / 3 I_{H_{\mathrm{V}}}$ & $1-6$ & N. A. & $\begin{array}{c}\text { Isotropic spherulite } \\
\text { size }\end{array}$ \\
\hline Winter $^{[37]}$ & iPP & $140-148$ & $I_{V_{\mathrm{V}}}$ for $I_{H_{\mathrm{V}}}=0$ & $0.1-2.8$ & 1 (max), 0.6-0.7 (final) & Size of fluctuations \\
\hline \multirow[t]{2}{*}{$\begin{array}{l}\text { Somwangthanaroj } \\
\text { et al. }{ }^{[49]}\end{array}$} & iPP & & & & $1(\max )$ & $\begin{array}{l}\text { Size of micron } \\
\text { scale structure }\end{array}$ \\
\hline & $\begin{array}{c}\mathrm{iPP}+ \\
\text { nanoclay }\end{array}$ & $140-150$ & $I_{V_{\mathrm{V}}}$ for $I_{H_{\mathrm{V}}}=0$ & $0.5-3.5$ & $\begin{array}{l}1 \text { (max) and } \\
\text { decreasing }\end{array}$ & $\begin{array}{l}\text { Size of clay aggl. or } \\
\text { blend structure size }\end{array}$ \\
\hline \multirow[t]{2}{*}{$\operatorname{Devaux}^{[41]}$} & iPP & $132-138$ & $I_{V_{V}}-4 / 3 I_{H_{V}}$ & $0.2-3$ & $0.3-2.2$ & Average distance \\
\hline & PVDF & 157 & $I_{V_{\mathrm{V}}}-4 / 3 I_{H_{\mathrm{V}}}$ & N. A. & 3 (max) 1.7 (final) & $\begin{array}{c}\text { between } \\
\text { dense zones }\end{array}$ \\
\hline Strobl ${ }^{[43]}$ & sPP & $103-123$ & $I_{H_{\mathrm{V}}}$ and $I_{V_{\mathrm{V}}}$ & $0.05-1$ & $1-5( \pm 10 \%)$ & $\begin{array}{c}\text { Hedrite size } \\
\left(H_{\mathrm{V}}\right) \text {, melt } \\
\text { filled holes }\left(V_{\mathrm{V}}\right)\end{array}$ \\
\hline Wang et al. ${ }^{\text {a) [55] }}$ & sPP & $115-240$ & $I_{V_{V}}$ & $0.5-2$ & $1.8 \pm 0.5$ & Domain width \\
\hline
\end{tabular}

a) Cold crystallization. 
even at early times, well before the upturn in $\underline{Q}_{\eta}$, the isotropic correlation length is large (about $1 \mu \mathrm{m}$ ), followed by a monotonic decrease over the course of the scattering experiment. According to the authors, this demonstrates that, at short times, when the magnitude of scattering is small because crystallite regions are only just beginning to form, isotropic features of size approximately $1 \mu \mathrm{m}$ exist in iPP nanocomposites and are thereby available to influence the subsequent crystallization kinetics. The group of Strob ${ }^{[43]}$ found that over the whole crystallization process of a sPP the scattering patterns for both $H_{\mathrm{V}}$ and $V_{\mathrm{V}}$ scattering were well represented by the Debye-Bueche structure factor. According to the authors, the correlation length derived from $H_{\mathrm{V}}$ scattering, $l_{\mathrm{HV}}$, provides a measure for the size of the hedrites. The correlation length derived from the $V_{\mathrm{V}}$ scattering, $l_{\mathrm{VV}}$, can be representative for either the hedrites or the melt-filled holes between them, depending on the degree of filling. At the early stage of crystallization $l_{\mathrm{HV}}$ and $l_{\mathrm{VV}}$ showed a steady increase with time due to the growth of the objects. However, with increasing time, fluctuations were observed in both correlation lengths. These came as expected for $l_{\mathrm{VV}}$, but they were also found for $l_{\mathrm{HV}}$, indicating a not ideally random orientation of crystals in the hedrites. They also found an increase in the final values of $l_{\mathrm{HV}}$ and $l_{\mathrm{VV}}$ with increasing $T_{\mathrm{c}}$. In the temperature range studied, the final value of $l_{\mathrm{HV}}$, giving the size of the hedrites, ranges from $1-5$ $\mu \mathrm{m}$. In his doctoral work, Devaux ${ }^{[41]}$ applied the DebyeBueche approach to $I_{V_{\mathrm{V}}}-\frac{4}{3} I_{H_{\mathrm{V}}}$, using two different approaches: subtraction of the thermal noise and subtraction of the contribution of the polymer melt. Both methods gave different results, especially in the early stages of crystallization. For quiescent crystallization of an iPP the correlation length increased with temperature (cf. Kawai et al. ${ }^{[42]}$ ) and its magnitude was of the same order as those calculated by Pogodina et al., ${ }^{[37]}$ that is, micron-size. However, in this case the data did not show an increase followed by a decrease. Rather, they showed a slight decrease as a function of time. For PVDF on the other hand, the correlation length first increased to a maximum, well before the maximum in $\underline{Q}_{\eta}$, and then decreased to a constant value of which the order of magnitude is again $1 \mu \mathrm{m}$. On the one hand, this is rather strange since the spherulites formed in PVDF are much smaller than those found in iPP. This underlines that the correlation length can not simply be correlated with the size of the semi- crystalline structures. Assuming the correlation length to be an average distance between dense zones and assuming that the dense zones are at the origin of the nuclei, this should result in micron-sized spherulites at the end of the crystallization. However, microscopic observations showed separation distances between nuclei much larger than the correlation lengths probed by the SALS experiments. There was a difference of two orders with reality, which suggests that not every dense zone results in an active nucleus. On the other hand, this observation is coherent with the knowledge of critical nuclei in the classical theories: only zones that attain a certain critical size become a nucleus. Wang et al. ${ }^{[55]}$ found that, prior to the cold crystallization of a sPP, the correlation length derived from the $V_{\mathrm{V}}$ patterns remains constant up to times corresponding with the increase in $Q_{\delta}$ and is about $1.13 \mu \mathrm{m}$, regardless of the crystallization temperature. They believe that $\xi$ is a characteristic length scale associated with the mean dimensions and volume fractions of the randomly distributed phases, and that the fact of unchanged $\xi$ implies a significantly limited coarsening of the phase-separated structure.

In our study on $\mathrm{iPB}$, the evolution of the correlation length of the density fluctuations was also probed. The Debye-Bueche analysis was applied to $I_{V_{\mathrm{V}}}-\frac{4}{3} I_{H_{\mathrm{V}}}$ (cfr. Okada et al. ${ }^{[35]}$ and Devaux ${ }^{[41]}$ ) and $I_{H_{\mathrm{V}}}$ (cfr. Okada et al. ${ }^{[35]}$ and the group of Strobl ${ }^{[43]}$ ) after subtracting the melt contribution. Since the model is only valid as long as no anisotropic elements appear, it should only be applied to $I_{V_{\mathrm{V}}}$ for $I_{H_{\mathrm{V}}}=0$ (cf. Pogodina et al. ${ }^{[37]}$ ). Nevertheless, in our experiments $H_{\mathrm{V}}$ scattering could be fitted similarly well (see Figure 9). We also believe that, by subtracting the contribution of anisotropic elements to isotropic scattering, the applicability of the Debye-Bueche theory is extended into the region where $Q_{\delta} \neq 0$. This is the first time correlation lengths are reported for flow-induced
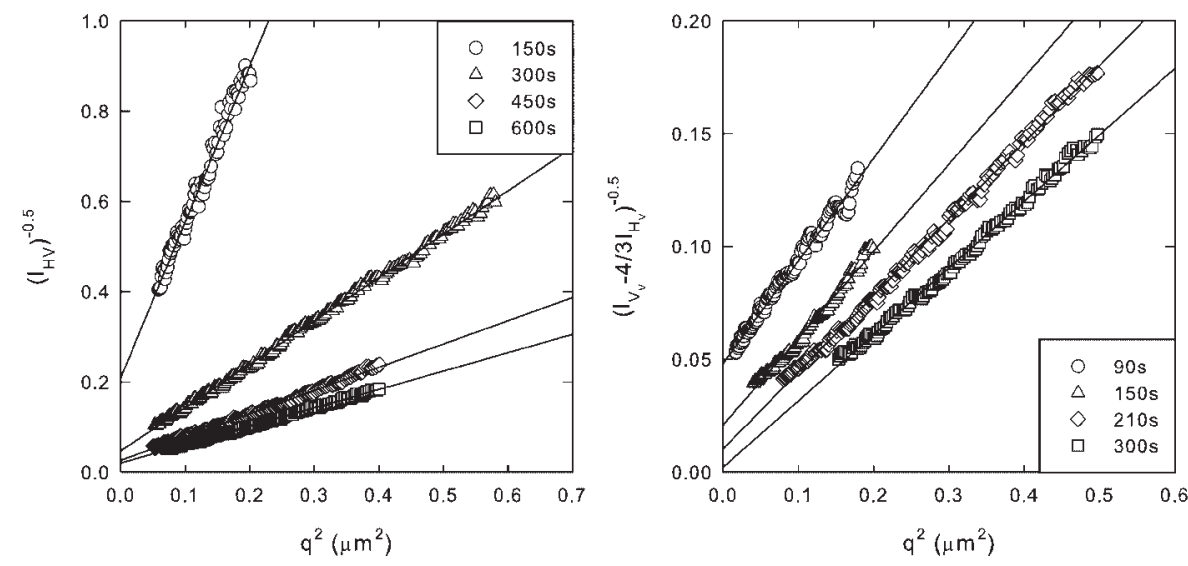

Figure 9. Debye-Bueche plots for $I_{H_{v}}$ (left) and $I_{V_{V}}-\frac{4}{3} I_{H_{v}}$ (right) during crystallization of PBo400 at $98^{\circ} \mathrm{C}$. Shearing conditions: $\dot{\gamma}=10 \mathrm{~s}^{-1}$ and $\gamma=100$. 
crystallization experiments. Although the Debye-Bueche analysis has been developed in the case of circularly symmetric scattering, the use of circularly averaged patterns can offer a solution in the cases where the scattering becomes anisotropic. Figure 9 shows that both $I_{H_{\mathrm{V}}}$ and $I_{V_{\mathrm{V}}}-\frac{4}{3} I_{H_{\mathrm{V}}}$ can be described by the Debye-Bueche scattering function.

Determination of the correlation lengths from the Debye-Bueche plots is not straightforward. First of all the practical use of these plots requires a limitation on the $q^{2}$ range for both small and large $q$, so that the plots show a linear behavior. In the current study this $q^{2}$ range is very narrow compared to literature values (see Figure 9 and Table 3). Also, the very small intercepts, which are used in the calculation of the correlation lengths [see Equation (10)], make it a very sensitive method: changing the $q^{2}$ range and/or omitting a few data points from the fit can already have a significant effect on the intercept and, thus, the correlation length. In some cases, the intercept can even be negative, which makes it impossible to calculate a correlation length. Our results did not show a clear trend in the time-evolution of the correlation length: in most cases the correlation lengths show a decrease to a constant value with time, although sometimes a maximum can also be seen. No obvious trends as a function of temperature, shear or molecular weight could be observed. For all experiments final values were in the range 1-10 $\mu \mathrm{m}$. In any case, the results demonstrate that the evaluation of the length scales of the observed phenomena by the Debye-Bueche analysis is not evident.

\section{SALS during Melting}

Melting of a fully crystallized structure is a convenient process for examining the theoretically predicted variations of the $H_{\mathrm{V}}$ and $V_{\mathrm{V}}$ invariants and it can also help in obtaining a better understanding of the concept of density fluctuations. According to Equation (6) and (8), a decrease in the absolute magnitude of $Q_{\delta}$ during melting can result from a decrease in $\phi_{\mathrm{s}}, \phi_{\mathrm{cr}, \mathrm{s}}$, and $f_{\mathrm{cr}, \mathrm{s}}$. However, during melting the number and size of the crystals are not expected to change because crystals do not remelt into nuclei but remain volume-filling and decrease in their crystalline content. Thus, the decrease in $Q_{\delta}$ upon melting corresponds to a reduction in crystallinity within the spherulites $\phi_{\mathrm{cr}, \mathrm{s}}$.

To the best of our knowledge, Akpalu et al. ${ }^{[52,53]}$ are the only group up to now that have monitored melt behavior using SALS. In a first paper, ${ }^{[52]}$ their goal was to measure the thermodynamic and structural variables necessary for describing the multiphase behavior of crystal-amorphous (PCL/PDLA) blends. They showed that the monotonic decrease in $Q_{\delta}$ is indeed caused by a decrease in $\phi_{c r, s}$
The temperature at which $Q_{\delta}$ reached zero was used as the final melting temperature of the crystals, and it agrees well with values obtained from DSC and WAXS measurements. For pure PCL the $V_{\mathrm{V}}$ invariant also decreased monotonically to the same melting temperature as that obtained from $H_{\mathrm{V}}$ scattering. Moreover, the $V_{\mathrm{V}}$ invariant remained constant after melting, indicating that there are no excess density fluctuations in the melt state once melting is complete. For the blend samples however, after complete melting of the PCL crystals, the $V_{\mathrm{V}}$ invariant initially showed a small upward slope, followed by a sharp increase. This sharp increase is characteristic of a lower critical solution temperature phase transition, arising from an increase in density fluctuations. In a subsequent paper ${ }^{[53]}$ SALS was used during melting of a homogeneous ethylene-1-hexene copolymer to determine the number of crystal populations, the melting temperature of each population, the spatial organization of crystalline aggregates, and their final melting temperature. The temperature variation of $H_{\mathrm{V}}$ and $V_{\mathrm{V}}$ patterns and integrated intensities during melting is consistent with predictions of a generalized SALS model. For a given crystallization condition, the final melting temperature obtained from $H_{\mathrm{V}}$ and $V_{\mathrm{V}}$ scattering is again in good agreement with values obtained from DSC and SAXS. Their results demonstrate that SALS can be used to understand and provide a quantitative description of how crystallization conditions affect the supermolecular structure organization in ethylene $/ \alpha$-olefin copolymers.

The goal of the melting experiments in this study was to see if, on heating the samples from the crystalline state, density fluctuations still exist after orientation fluctuations have disappeared. It is important to mention that in this paper the density invariant $Q_{\eta}$ is used, as opposed to the $V_{\mathrm{V}}$ invariant, $Q_{V_{\mathrm{V}}}$, in the work of Akpalu et al. ${ }^{[52,53]}$ However, $Q_{V_{V}}$ can be easily calculated combining Equation (3) and (4):

$$
Q_{V_{\mathrm{v}}}=\int_{0}^{\infty} I_{V_{\mathrm{v}}} q^{2} \mathrm{~d} q=\underline{Q}_{\eta}+\frac{4}{3} \underline{Q}_{\delta}
$$

Figure 10 shows the evolution of $Q_{\delta}, Q_{\eta}$ and $Q_{V_{\mathrm{V}}}$ upon melting at $2^{\circ} \mathrm{C} \cdot \mathrm{min}^{-1}$ from the crystalline state obtained after shearing PB0400 $\left(\gamma=10 s^{-1}, \gamma=10\right)$ before isothermal crystallization at $98^{\circ} \mathrm{C}$. The plotted values are absolute values. The observation is similar to that obtained for pure PCL by Akpalu et al.: ${ }^{[52]}$ both $Q_{\delta}$ and $Q_{V_{\mathrm{V}}}$ show a monotonic decrease, and the latter remains constant after melting, indicating that there are no excess density fluctuations in the melt state. Despite the fact that $Q_{\delta}$ decreases faster than $Q_{V_{V}}$ and that $Q_{V_{V}}$ is still quite large when $Q_{\delta}$ is almost zero, the instants when both invariants reach exactly zero 


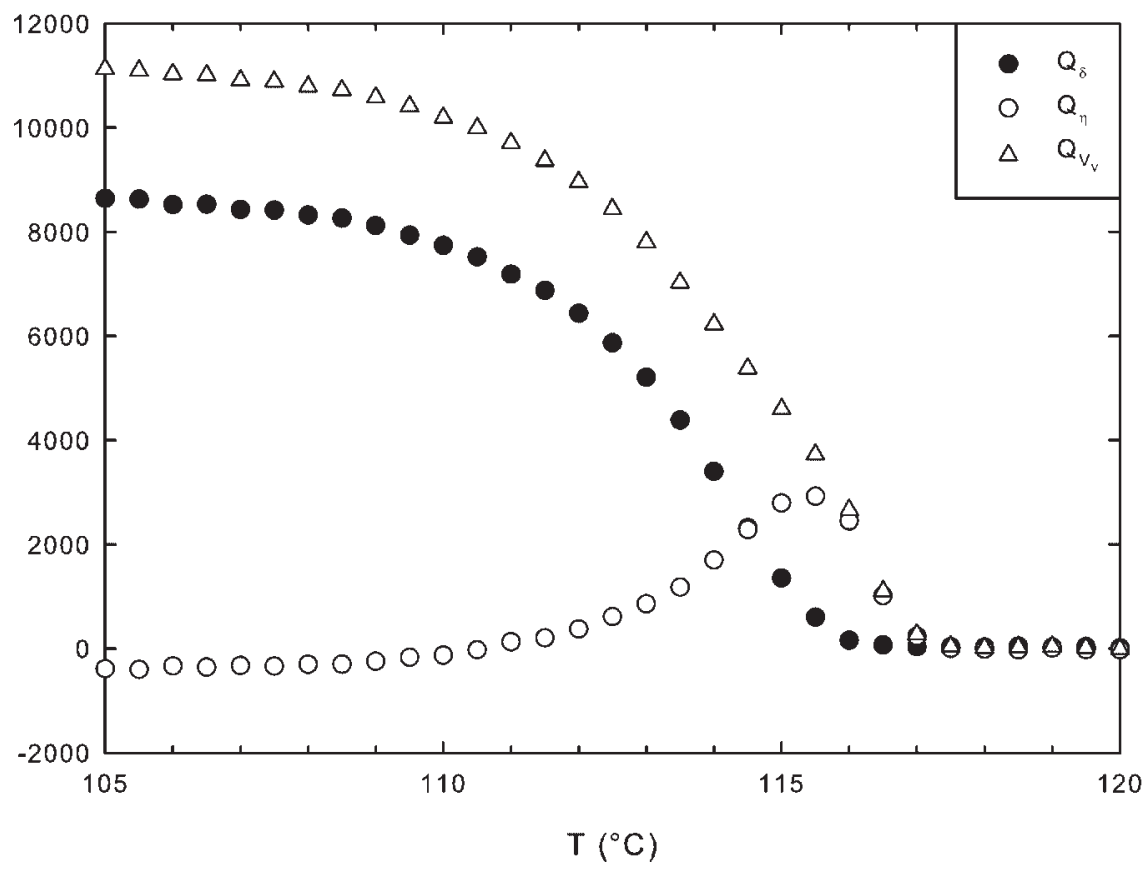

Figure 10. Evolution of the invariants upon melting at $2{ }^{\circ} \mathrm{C} \cdot \min ^{-1}$ from the crystalline state obtained after crystallization of PBO400 at $98^{\circ} \mathrm{C}$. Shearing conditions: $\dot{\gamma}=10 \mathrm{~s}^{-1}$ and $\gamma=10$.

agree within the time resolution of the measurements, which is again in accordance with the observation by Akpalu et al. ${ }^{[52]}$

\section{Conclusion}

In the present work, the fact that nucleation might be accompanied by large scale density fluctuations was investigated for the flow-induced crystallization of iPB. To understand what controls the structure formation in semi-crystalline polymers and to exploit the effects of flow on the early stages in more detail, SALS has been combined with POM. The evolution of the scattering patterns was described in terms of the invariants $\underline{Q}_{\eta}$ and $Q_{\delta}$ that are an expression of the growing fluctuations of density and orientation respectively. The invariants showed the typical behavior for polymer crystallization and the observed difference in onset time between both was explained in terms of the development of weakly anisotropic structures in the early stages of crystallization. The combination with POM enabled the results to be linked with the developing semi-crystalline structures and confirmed that density fluctuations, at least at the instant they are detected with SALS, are an indication of the presence of weakly anisotropic structures. It was also shown that the use of correlation lengths derived from the Debye-Bueche analysis of the SALS intensities is delicate and that it is difficult to link the results with the developing semi-crystalline structures. Furthermore, SALS experiments were carried out during melting of the crystallized structure to verify whether, on heating the samples from the crystalline state, density fluctuations still exist after orientation fluctuations have disappeared, which was not the case. For both crystallization and melting experiments, the observations suggest that the detected density fluctuations result from the presence of weakly anisotropic domains, rather than being an indication of densification before the onset of crystallization. These weakly anisotropic domains are loosely structured spherulites for which the crystalline parts are too tiny to cause a measurable anisotropy by themselves. With ongoing crystallization, the internal structure of these spherulites matures, and orientation fluctuations caused by the growing anisotropic entities also start to play a role. In fact, these conclusions accord with the results of a recent paper by Xiao et al., ${ }^{[56]}$ which showed that the early stage crystallization is characterized by the formation of anisotropic domains before any local crystalline structure can be detected, followed by orientational ordering of the domains. Our results are thus in favour of a nucleation and growth mechanism for the early stages of the flow-induced crystallization of iPB, at least within the range of experimental conditions investigated.

Received: December 14, 2007 Revised: January 30, 2008; Accepted: January 31, 2008; DOI: 10.1002/mame.200700407

Keywords: density fluctuations; flow-induced crystallization; light scattering

[1] J. I. Lauritzen, J. D. Hoffman, J. Res. Natl. Bur. Stand., Sect. A 1960, 64, 73.

[2] G. Hauser, J. Schmidtke, G. Strobl, Macromolecules 1998, 31, 6250.

[3] G. Strobl, Eur. Phys. J. E 2000, 3, 165.

[4] P. D. Olmsted, W. C. K. Poon, T. C. B. McLeish, N. J. Terrill, A. Ryan, Phys. Rev. Lett. 1998, 81, 373.

[5] K. Katayama, Sen'i Gakkaishi 1967, 23, S300.

[6] K. Katayama, T. Amano, K. Nakamura, Kolloid Z. Z. Polym. 1968, 226, 125.

[7] G. S. Yeh, P. H. Geil, J. Macromol. Sci., Part B: Phys. 1967, 1, 235.

[8] P. H. Lindenmeyer, Polym. Eng. Sci. 1974, 14, 456. 
[9] G. Allegra, J. Chem. Phys. 1977, 12, 5453.

[10] G. Allegra, S. V. Meille, Phys. Chem. Chem. Phys. 1999, 1, 5179.

[11] J. M. Schultz, J. S. Lin, R. W. Hendricks, J. Petermann, R. M. Gohil, J. Polym. Sci., Part B: Polym. Phys. 1981, 19, 609.

[12] M. Imai, K. Mori, T. Mizukami, K. Kaji, T. Kanaya, Polymer 1992, 33, 4451.

[13] M. Imai, K. Mori, T. Mizukami, K. Kaji, T. Kanaya, Polymer 1992, 33, 4457.

[14] M. Imai, K. Kaji, T. Kanaya, Macromolecules 1994, 27, 7103.

[15] M. Imai, K. Kaji, T. Kanaya, Y. Sakai, Phys. Rev. B 1995, 52, 12696.

[16] T. A. Ezquerra, E. LopezCabarcos, B. S. Hsiao, F. J. BaltaCalleja, Phys. Rev. E 1996, 54, 989.

[17] E. L. Heeley, C. K. Poh, W. Li, A. Maidens, W. Bras, I. P. Dolbnya, A. J. Gleeson, N. J. Terrill, P. A. Fairclough, P. D. Olmsted, R. I. Ristic, J. Hounslow, A. J. Ryan, Faraday Discuss. Chem. Soc. 2003, 122, 343.

[18] E. L. Heeley, A. Maidens, P. D. Olmsted, W. Bras, I. P. Dolbnya, P. A. Fairclough, N. J. Terrill, A. J. Ryan, Macromolecules 2003, 36, 3656.

[19] Z. G. Wang, B. S. Hsiao, E. B. Sirota, S. Srinivas, Polymer 2000, $41,8825$.

[20] K. Kaji, K. Nishida, G. Matsuba, T. Kanaya, M. Imai, J. Macromol. Sci., Part B: Phys. 2003, 42, 709.

[21] M. Imai, K. Kaji, T. Kanaya, Phys. Rev. Lett. 1993, 71, 4162.

[22] G. Matsuba, K. Kaji, K. Nishida, T. Kanaya, M. Imai, Macromolecules 1999, 32, 8932.

[23] G. Matsuba, K. Kaji, K. Nishida, T. Kanaya, M. Imai, Polym. J. 1999, 31, 722

[24] F. M. Mirabella, J. Polym. Sci., Part B: Polym. Phys. 2006, 44, 2369.

[25] Y. Akpalu, E. J. Amis, J. Chem. Phys. 1999, 111, 8686.

[26] Z. G. Wang, B. S. Hsiao, E. B. Sirota, P. Agarwal, S. Srinivas, Macromolecules 2000, 33, 978.

[27] M. Hikosaka, K. Watanabe, K. Okada, S. Yamazaki, Adv. Polym. Sci. 2005, 191, 137.

[28] C. Liu, M. Muthukumar, J. Chem. Phys. 1998, 109, 2536.

[29] M. Muthukumar, Eur. Phys. J. E 2000, 3, 199.

[30] B. Lotz, Eur. Phys. J. E 2000, 3, 185.

[31] B. Lotz, Adv. Polym. Sci. 2005, 180, 17.

[32] R. S. Stein, P. R. Wilson, J. Appl. Phys. 1962, 33, 1914.

[33] D. Y. Yoon, R. S. Stein, J. Polym. Sci., Polym. Phys. Ed. 1974, 12, 735.

[34] J. T. Koberstein, T. Russel, R. S. Stein, J. Polym. Sci., Polym. Phys. Ed. 1979, 17, 1719.

[35] T. Okada, H. Saito, T. Inoue, Macromolecules 1992, 25, 1908.

[36] M. Imai, K. Kaji, Polymer 2006, 47, 5544.

[37] N. V. Pogodina, S. K. Siddiquee, J. W. van Egmond, H. H. Winter, Macromolecules 1999, 32, 1167.
[38] N. V. Pogodina, V. P. Lavrenko, S. Srinivas, H. H. Winter, Polymer 2001, 42, 9031.

[39] A. Elmoumni, H. H. Winter, A. J. Waddon, H. Fruitwala, Macromolecules 2003, 36, 6453.

[40] N. Devaux, B. Monasse, J. Haudin, P. Moldenaers, J. Vermant, Rheol. Acta 2004, 43, 210.

[41] N. Devaux, Ph. D. Thesis, Sophia-Antipolis 2003.

[42] H. Janeschitz-Kriegl, Macromolecules 2006, 39, 4448.

[43] T. Kawai, G. Strobl, Macromolecules 2004, 37, 2249.

[44] Y. M. Mao, O. Yang, G. Li, Y. Huang, K. Yang, J. Macromol. Sci., Part B: Phys. 2005, 44, 353.

[45] Y. Ogino, H. Fukushima, G. Matsuba, N. Takahashi, K. Nishida, T. Kanaya, Polymer 2006, 47, 5669.

[46] H. Fukushima, Y. Ogino, G. Matsuba, K. Nishida, T. Kanaya, Polymer 2005, 46, 1878.

[47] Y. Ogino, H. Fukushima, N. Takahashi, G. Matsuba, K. Nishida, T. Kanaya, Macromolecules 2006, 39, 7617.

[48] C. Zhang, H. Hu, X. Wang, Y. Yao, X. Dong, D. Wang, Z. Wang, C. Han, Polymer 2007, 48, 1105.

[49] A. Somwangthanaroj, E. C. Lee, M. J. Solomon, Macromolecules 2003, 36, 2333.

[50] Z. Xiao, Y. Li, D. Ma, L. S. Schadler, Y. Akpalu, J. Polym. Sci., Part B: Polym. Phys. 2006, 44, 1084.

[51] Y. Akpalu, L. Kielhorn, B. S. Hsiao, R. S. Stein, T. P. Russell, J. van Egmond, M. Muthukumar, Macromolecules 1999, 32, 765.

[52] Y. Akpalu, Y. Lin, J. Polym. Sci., Part B: Polym. Phys. 2002, 40 2714.

[53] Y. Li, Y. Akpalu, Macromolecules 2004, 37, 7265.

[54] K. Nishida, K. Kaji, T. Kanaya, G. Matsuba, T. Konishi, J. Polym. Sci. 2004, 42, 1817.

[55] C. Wang, C. Lin, C. Chu, Polymer 2005, 46, 12595.

[56] Z. Xiao, Y. Akpalu, Polymer 2007, 48, 5388.

[57] J. Baert, P. Van Puyvelde, Polymer 2006, 47, 5871.

[58] J. Baert, P. Van Puyvelde, F. Langouche, Macromolecules 2006, 39, 9215.

[59] P. Debye, A. M. Bueche, J. Appl. Phys. 1949, 20, 518.

[60] J. M. Haudin, "Optical studies of polymer morphology", in Optical properties of polymers, G. H. Meeten, Ed., Elsevier Applied Science, Barking 1986, p. 167-264.

[61] R. S. Stein, G. L. Wilkes, "Physico-Chemical Approaches to the Measurement of Anisotropy and Orientation", in: Structure and Properties of Oriented Polymers, $1^{\text {st }}$ Ed., I. M. Ed., Applied Science Publishers, London 1975, p. 85.

[62] Yoon, D. Y. Ward, R. S. Stein, J. Polym. Sci. Polym. Phys. Ed. $1974,12,763$

[63] H. C. van de Hulst, "Light Scattering by Small Particles", $2^{\text {nd }}$ Ed., Dover Publications Inc., New York 1981, p. 37. 